\title{
Monotone Numerical Schemes for a Dirichlet Problem for Elliptic Operators in Divergence Form
}

\author{
Nedžad Limić * and Mladen Rogina ${ }^{\dagger}$
}

November 13, 2018

\begin{abstract}
We consider a second order differential operator $A(\boldsymbol{x})=-\sum_{i, j=1}^{d} \partial_{i} a_{i j}(\boldsymbol{x}) \partial_{j}+$ $\sum_{j=1}^{d} \partial_{j}\left(b_{j}(\boldsymbol{x}) \cdot\right)+c(\boldsymbol{x})$ on $\mathbb{R}^{d}$, on a bounded domain $D$ with Dirichlet boundary conditions on $\partial D$, under mild assumptions on the coefficients of the diffusion tensor $a_{i j}$. The object is to construct monotone numerical schemes to approximate the solution to the problem $A(\boldsymbol{x}) u(\boldsymbol{x})=\mu(\boldsymbol{x}), \quad \boldsymbol{x} \in D$, where $\mu$ is a positive Radon measure. We start by briefly mentioning questions of existence and uniqueness, introducing function spaces needed to prove convergence results. Then, we define non-standard stencils on grid-knots that lead to extended discretization schemes by matrices possesing compartmental structure. We proceed to discretization of elliptic operators, starting with constant diffusion tensor and ending with operators in divergence form. Finally, we discuss $W_{2}^{1}$-convergence in detail, and mention convergence in $C$ and $L_{1}$ spaces. We conclude by a numerical example illustarting the schemes and convergence results.
\end{abstract}

Key words: Elliptic operator, divergence form, monotone scheme

AMS subject classification: (2000) 35J20, 35J25, 35J15, 65N06, 65N15

\section{Introduction}

The object of present analysis are numerical solutions of the elliptic boundary value problems in terms of monotone schemes. It is assumed that the elliptic differential operator has the divergence form with measurable coefficients satisfying the strict ellipticity condition. The basic idea of construction of monotone schemes is presented in [MW] without analysis of convergence of approximate solutions. Some elaborations of this basic idea can be found in SMMM, LR2 and [LR3 where the convergence is considered in $C$ - and $L_{1}$-spaces. In these works the stencils of schemes are enclosed by rectangles with vertices at grid-knots. In the present work we extend the published results by constructing schemes with stencils stretching far from basic grid-rectangles and so being conceptually closer to the original idea of [MW]. The schemes are not derived from finite difference operators approximating differential operators but rather from a general principle which ensures the convergence of approximate solutions. For the case of a classical elliptic problem this general principle is necessary and sufficient for the convergence in Hölder spaces.

${ }^{*}$ Dept. of Mathematics, University of Zagreb, Bijenička 30, 10002 Zagreb, Croatia, e-mail: nlimic@math.hr

${ }^{\dagger}$ Dept. of Mathematics, University of Zagreb, Bijenička 30, 10002 Zagreb, Croatia, e-mail: rogina@math.hr 
In Section 2 we describe boundary value problems for linear elliptic differential operators which are analyzed here from the numerical point of view. Problems for bounded domains and Dirichlet boundary conditions are our main interest. For the sake of completeness, numerical methods for problems on the whole real space are also studied.

Monotone schemes for linear elliptic problems can be easily constructed by using discretizations of the corresponding differential operators in terms of matrices of positive type. Therefore, in Section 3 we start our analysis by defining matrices of positive type and matrices with the compartmental structure. The latter ones are more pertinent to construction of discretizations of elliptic differential operators in divergence form. Monotone schemes give rise to grid-solutions. By embedding gridfunctions into the space of hat functions we pass from grid-solutions to functions which are called approximate solutions. The remaining part of this section contains some technical results relating various norms of grid-solutions and the corresponding norms of approximate solutions.

At the beginning of Section 4 we describe discretizations of the operator $A=$ $-\sum a_{i j} \partial_{i} \partial_{j}$ in terms of matrices of positive type by using forward and backward finite difference operators LR1. Then we describe another method of discretization which is not based on any kind of finite difference operators. This method becomes a basis for discretizations of the elliptic operators in divergence form, $A=-\sum \partial_{i} a_{i j} \partial_{j}$. The resulting schemes are called extended schemes. The strong ellipticity of associated discretized bilinear forms $[\mathrm{YO}$ is analyzed and proved for the class of extended schemes.

The convergence of numerical solutions is analyzed in Section 5 , First we consider the case of $W_{2}^{1}$-convergence and later the convergence in Hölder spaces and $L_{1}$-spaces. The convergence in $W_{2}^{1}$-space is proved by using the standard finite element techniques.

Some technical problems arising from a non-continuity of the functions $a_{i j}, i \neq j$, are discussed in Section 6. It is demonstrated by an example how the extended schemes can be applied straightforwardly to problems with general measurable functions $a_{i j}$.

\section{Preliminaries}

Elements of $\mathbb{R}^{d}$ are denoted by $\boldsymbol{x}, \boldsymbol{w}, \boldsymbol{r}$ etc. The Euclidean norm in $\mathbb{R}^{d}$ is denoted by $|\cdot|$. For a subset $S \subset \mathbb{R}^{d}$ the closure is denoted by $\bar{S}$ or $\operatorname{cls}(S)$ and boundary by $\partial S$ or $\operatorname{bnd}(S)$. For an open set $D$ the scalar product and norm of $L_{2}(D)$ are denoted by $(\cdot \mid \cdot)$ and $\|\cdot\|_{2}$, respectively. The norm of $L_{p}(D)$ is denoted by $\|\cdot\|_{p}$. The Sobolev $W_{p}^{1}$-spaces and $\dot{W}_{p}^{1}$-spaces are defined in a standard way [Ma, St]. In addition we need the Hölder spaces $C^{(\alpha)}(\bar{D}), \alpha \in(0,1)$, defined as in $\mathrm{Ma}$, St. The convex set of positive Radon measures $\mu$ on $\mathfrak{B}(D), \int d \mu=1$ (tight probability measures) is denoted by $\mathcal{P}(D)$. Then $\langle v \mid \mu\rangle=\int_{D} v(\boldsymbol{x}) \mu(d \boldsymbol{x})$ is well defined for $v \in \dot{W}_{\infty}^{1}(D)$. We say that a sequence of $\mu_{n} \in \mathcal{P}(D)$ converges weakly to $\mu \in \mathcal{P}(D)$ if $\lim _{n}\left\langle v \mid \mu_{n}\right\rangle=\langle v \mid \mu\rangle$ for each $v \in \dot{C}(\bar{D})$. An open subset of $\mathbb{R}^{d}$ with Lipshitz boundary [St] is called a domain with Lipshitz boundary. Only such domains are considered here. The following result is used. Let $\dot{C}^{(1)}(\bar{D})$ be the linear space of continuous functions, having continuous partial derivatives and zero values on $\partial D$. Then, for a domain $D$ with Lipshitz boundary, the space $\dot{C}^{(1)}(\bar{D})$ is dense in $\dot{W}_{p}^{1}(D), 1 \leq p<\infty$ Ma].

Here we study elliptic operators on $\mathbb{R}^{d}$ in divergence form defined by

$$
A(\boldsymbol{x})=-\sum_{i, j=1}^{d} \partial_{i} a_{i j}(\boldsymbol{x}) \partial_{j}+\sum_{j=1}^{d} \partial_{j}\left(b_{j}(\boldsymbol{x}) \cdot\right)+c(\boldsymbol{x}),
$$


for which the coefficients are constrained as follows. The functions $a_{i j}=a_{j i}, b_{i}, i, j=$ $1,2, \ldots, d$ and $c$ are measurable on $\mathbb{R}^{d}, c \geq 0$ and $a_{i j}(\boldsymbol{x})$ converge to constant values as $|\boldsymbol{x}|$ increases. Apart from this, the elliptic operator must be strictly elliptic, meaning that there are positive numbers $\underline{M}, \bar{M}, 0<\underline{M} \leq \bar{M}$, such that the double inequality

$$
\underline{M}|\boldsymbol{x}|^{2} \leq \sum_{i, j=1}^{d} a_{i j}(\boldsymbol{x}) z_{i} \bar{z}_{j} \leq \bar{M}|\boldsymbol{x}|^{2}, \quad \boldsymbol{x} \in \mathbb{R}^{d},
$$

holds. The main part of $A$ is denoted by $A_{0}$.

For sufficiently large and positive $\lambda$ there exist kernels $(\boldsymbol{x}, \boldsymbol{y}) \mapsto K_{A}(\lambda, \boldsymbol{x}, \boldsymbol{y})$ on $D \times D$, such that $(\boldsymbol{x}, \boldsymbol{y}) \mapsto|\boldsymbol{x}-\boldsymbol{y}|^{d-2} K_{A}(\lambda, \boldsymbol{x}, \boldsymbol{y})$ are uniformly bounded on $D \times D$ and Hölder continuous in $\boldsymbol{x}$ on the set $D \backslash \boldsymbol{y}$. The integral operator with kernel $K_{A}(\lambda, \cdot, \cdot)$ is denoted by $R(\lambda, A)$. It maps measurable and bounded functions with compact supports into continuous functions on $D$ and $(\lambda I+A) R(\lambda, A)=I$ in $C(\bar{D})$. Let $\mathfrak{L}(D)$ be the linear space of functions on $D$ and $\mathfrak{D}\left(A_{D}\right)=R(\lambda, A) \mathfrak{L}(D)$. Then $A(\boldsymbol{x}): \mathfrak{D}\left(A_{D}\right) \mapsto \mathfrak{L}(D)$ is denoted by $A_{D}$. We say that $A_{D}$ is defined by $A(\boldsymbol{x})$ on $D$ with Dirichlet boundary conditions on $\partial D$. If $\mathfrak{L}(D)=L_{p}(D), p<\infty$, the operator $A_{D}$ is maximally closed from $\mathfrak{D}\left(A_{D}\right) \subset L_{p}(D)$ into $L_{p}(D)$.

Let us define a real bilinear form on $W_{q}^{1}(D) \times W_{p}^{1}(D), 1 / p+1 / q=1$, by:

$$
\begin{gathered}
a(v, u)=\sum_{i, j=1}^{d} \int_{D} a_{i j}(\boldsymbol{x}) \partial_{i} v(\boldsymbol{x}) \partial_{j} u(\boldsymbol{x}) d \boldsymbol{x} \\
-\sum_{i=1}^{d} \int_{D} b_{i}(\boldsymbol{x}) \partial_{i} v(\boldsymbol{x}) u(\boldsymbol{x}) d \boldsymbol{x}+\int_{D} c(\boldsymbol{x}) v(\boldsymbol{x}) u(\boldsymbol{x}) d \boldsymbol{x} .
\end{gathered}
$$

For a domain $D$ with Lipshitz boundary $\partial D$ and for each pair $v \in \dot{W}_{q}^{1}(D), u \in$ $\dot{W}_{p}^{1}(D) \cap\left\{A u \in\left(\dot{W}_{q}^{1}(D)\right)^{\dagger}\right\}, 1<p<\infty$, there holds the Green's formula:

$$
a(v, u)=\langle v \mid A u\rangle \text {. }
$$

The Green's formula is also valid for each pair $v \in \dot{W}_{\infty}^{1}(D), u \in \dot{W}_{1}^{1}(D) \cap\{A u \in$ $\mathcal{P}(D)\}$.

The boundary value problem, to be studied in this work, is defined by

$$
\begin{gathered}
A(\boldsymbol{x}) u(\boldsymbol{x})=\mu(\boldsymbol{x}), \quad \boldsymbol{x} \in D, \\
u \mid \partial D=0,
\end{gathered}
$$

where $D$ is a domain with Lipshitz boundary and the nonhomogeneous term $\mu$ is either a positive Radon measure or $\mu \in \dot{W}_{2}^{-1}(D)$. In the case of $D=\mathbb{R}^{d}$ we understand that the boundary condition in (4) is omitted.

The variational formulation of (4) for a solution $u \in \dot{W}_{p}^{1}(D), p=1,2$, has the following form:

$$
a(v, u)=\langle v \mid \mu\rangle, \quad \text { for any } v \in \dot{W}_{q}^{1}(D) .
$$

Solutions of (4) and (5) are called strong and weak solutions, respectively.

The following result is valid [LR2].

THEOREM 2.1 Let $D$ be a bounded domain with Lipshitz boundary. For each $p \in$ $[1, d /(d-1))$ there exists a unique weak solution u of (5) belonging to the class $\dot{W}_{p}^{1}(D)$ and possessing the following properties:

(i) There exists a positive number $c$ depending on $\underline{M}, \bar{M}, p, D$, such that the following inequality is valid:

$$
\|u\|_{p, 1}<c .
$$

(ii) If $\left\{\mu_{n}: n \in \mathbb{N}\right\} \subset \mathcal{P}(D)$ converges weakly to a $\mu \in \mathcal{P}(D)$, then the corresponding sequence of weak solutions $\left\{u_{n}: n \in \mathbb{N}\right\} \subset \dot{W}_{p}^{1}(D), u_{n}=A_{D}^{-1} \mu_{n}$, converges strongly in $L_{p}(D)$ to $u=A_{D}^{-1} \mu$. 


\section{Grid-functions and $W_{2}^{1}$-spaces}

Let the orthogonal coordinate system in $\mathbb{R}^{d}$ be determined by unit vectors $\boldsymbol{e}_{i}$, and let us, for each $n \in \mathbb{N}$, define a numerical grid $G_{n}$ on $\mathbb{R}^{d}$ by vectors $\boldsymbol{x}=\sum_{l=1}^{d} h(n) k_{l} \boldsymbol{e}_{l}$, where the grid-step $h(n)$ is determined by $h(n)=2^{-n}$. If not necessary the gridsteps $h(n)$ are shortly denoted by $h$. For the sake of simple notation the grid step $h$ is assumed to be the same for all coordinate directions. The obtained results of convergence are valid as well for grids with grid-steps depending on direction $\boldsymbol{e}_{i}$. To each $\boldsymbol{v} \in G_{n}$ there corresponds a grid-cube $C(h, \boldsymbol{v})=\prod_{1}^{d}\left[v_{j}, v_{j}+h\right)$, where $v_{j}$ are coordinates of $\boldsymbol{v}$. Cubes $C(h, \boldsymbol{v})$ define a decomposition of $\mathbb{R}^{d}$ into disjoint sets. We say that the sets $G_{n}(D)=G_{n} \cap D$ are discretizations of $D$. The grids $G_{n}$ are homogeneous with respect to translations in the direction of coordinate axes, i.e. for $\boldsymbol{x} \in G_{n}$ and $\boldsymbol{t}=h p_{i} \boldsymbol{e}_{i}, p_{i} \in \mathbb{Z}$ we have $\boldsymbol{x}+\boldsymbol{t} \in G_{n}$. There exist subsets of $G_{n}$ which are also homogeneous in the defined sense. Let $\boldsymbol{r}_{0} \in G_{n}$ and $\boldsymbol{r}=\left(r_{1}, r_{2}, \ldots, r_{d}\right) \in \mathbb{N}^{d}$ be fixed. The set

$$
G_{n}\left(\boldsymbol{r}_{0}, \boldsymbol{r}\right)=\left\{\boldsymbol{r}_{0}+h \sum_{l=1}^{d} k_{l} r_{l} \boldsymbol{e}_{l}: k_{l} \in \mathbb{Z}\right\}
$$

is a subsets of $G_{n}$. It is homogeneous with respect to translations for $h k_{i} r_{i} \boldsymbol{e}_{i}, k_{i} \in \mathbb{Z}$, grid-knots. There are $\operatorname{vol}(R)=\prod_{i=1}^{d} r_{i}$ disjoint subgrids (6) making a partition of $G_{n}$. Each is denoted $G_{n}(R)$, where $R$ stands shortly for $2 d$ parameters $\boldsymbol{r}_{0} \in G_{n}, \boldsymbol{r} \in \mathbb{N}^{d}$. The index set of $G_{n}$ is denoted by $I_{n}$. Similarly, $I_{n}(R)$ is the index set of $G_{n}(R)$.

The discretization of a function $u \in C\left(\mathbb{R}^{d}\right)$ on $G_{n}$ is denoted by $\mathbf{u}_{n}$ and defined by values at grid-knots, $\left(\mathbf{u}_{n}\right)_{m}=u\left(\boldsymbol{x}_{\boldsymbol{m}}\right)$ where $\boldsymbol{x}_{\boldsymbol{m}}=\left(m_{1} h, m_{2} h, \ldots, m_{d} h\right) \in G_{n}$, and $\boldsymbol{m}=\left(m_{1}, m_{2}, \ldots, m_{d}\right)$ is a multi-index. The function $\mathbf{u}_{n}$ is usually called a grid function. We denote the linear spaces of discretizations by $l\left(G_{n}\right)$ or $l\left(G_{n}(D)\right)$. Elements of $l\left(G_{n}\right)$ are also called columns. The corresponding $L_{p}$-spaces are denoted by $l_{p}\left(G_{n}\right)$ or $l_{p}\left(G_{n}(D)\right)$, and their norms by $|\cdot|_{p}$. The duality pairing of $\mathbf{v} \in l_{q}\left(G_{n}\right)$ and $\mathbf{u} \in l_{p}\left(G_{n}\right)$ is denoted by $\langle\mathbf{v} \mid \mathbf{u}\rangle$. The scalar product in $l_{2}\left(G_{n}\right)$ is denoted by $\langle\cdot \mid \cdot\rangle$ and sometimes by $(\cdot \mid \cdot)$. The norm of $l_{p}\left(G_{n}(R)\right)$ is denoted by $|\cdot| R p$. For $p \in[1, \infty)$ we have

$$
|\mathbf{u}|_{R p}=\left[\operatorname{vol}(R) \sum_{\boldsymbol{k} \in I_{n}(R)}\left|u_{\boldsymbol{k}}\right|^{p}\right]^{1 / p},
$$

while $|\mathbf{u}|_{R \infty}=\sup \left\{\left|u_{\boldsymbol{k}}\right|: \boldsymbol{k} \in I_{n}(R)\right\}$. A matrix $A_{n}$ with indices corresponding to grid-knots of $G_{n}$ (or $G_{n}(D)$ ) is said to be defined on $G_{n}$ (or $G_{n}(D)$ ). We use the notation $A_{n} \geq 0$ if all entries of $A_{n}$ are non negative.

The shift operator $Z(\boldsymbol{x}), \boldsymbol{x} \in \mathbb{R}^{d}$, acting on functions $f: \mathbb{R}^{d} \mapsto \mathbb{R}$, is defined by $(Z(\boldsymbol{x}) f)(\boldsymbol{x})=f(\boldsymbol{x}+\boldsymbol{z})$. Similarly we define the discretized shift operator by $\left(Z_{n}(r, i) \mathbf{u}_{n}\right)_{\boldsymbol{m}}=\left(\mathbf{u}_{n}\right)_{\boldsymbol{n}}$, where $\boldsymbol{n}=\boldsymbol{m}+r h \boldsymbol{e}_{i}$.

Discretization of differential operators. A function $u \in C^{(1)}\left(\mathbb{R}^{d}\right)$ has continuous partial derivatives $\partial_{i} u, i=1,2, \ldots d$. With respect to a grid step $h$, the partial derivatives are discretized by forward/backward finite difference operators in the usual way:

$$
\begin{aligned}
& \mathrm{a}_{i}(t) u(\boldsymbol{x})=\frac{1}{t}\left(u\left(\boldsymbol{x}+t \boldsymbol{e}_{i}\right)-u(\boldsymbol{x})\right), \quad \\
& \widehat{\mathrm{U}}_{i}(t) u(\boldsymbol{x})=\frac{1}{t}\left(u(\boldsymbol{x})-u\left(\boldsymbol{x}-t \boldsymbol{e}_{i}\right)\right) .
\end{aligned}
$$

Discretizations of the functions $\partial_{i} u$ on $G_{n}$, denoted by $U_{i}(r) \mathbf{u}_{n}, V_{i}(r) \mathbf{u}_{n}$, are defined by:

$$
\left(U_{i}(r) \mathbf{u}_{n}\right)_{\boldsymbol{m}}=\Xi_{i}(r h) u\left(\boldsymbol{x}_{\boldsymbol{m}}\right), \quad\left(V_{i}(r) \mathbf{u}_{n}\right)_{\boldsymbol{m}}=\widehat{\mathrm{a}}_{i}(r h) u\left(\boldsymbol{x}_{\boldsymbol{m}}\right) .
$$

Then

$$
\begin{aligned}
& U_{i}(r)=(r h)^{-1}\left(Z_{n}(r, i)-I\right), \\
& V_{i}(r)=(r h)^{-1}\left(I-Z_{n}(-r, i)\right)=U_{i}(-r)=-U_{i}(r)^{T} .
\end{aligned}
$$


Therefore we have $U_{i}(-r)=U_{i}(r) Z_{n}(-r, i)=Z_{n}(-r, i) U_{i}(r)$, and similarly for $V_{i}(r)$.

In accordance with the previous terminology, we say that $\partial_{i}, \sum_{i j} \partial_{i} a_{i j} \partial_{j}$ etc. are differential operators on $\mathbb{R}^{d}$ or $D$. We say that their discretizations are defined on $G_{n}$ or $G_{n}(D)$. In particular, discretizations of the differential operator (1) are denoted by $A_{n}$. Naturally, matrices $A_{n}$ are the main object in this work. We intend to analyze a class of discretizations $A_{n}$ of (1) with compartmental structure.

DEFINITION 3.1 (Compartmental structure) A matrix $A=\left\{a_{i j}\right\}_{I I}$ is said to have the compartmental structure if it has positive diagonal entries, non-positive off-diagonal entries and positive or zero column sums:

$$
a_{i i} \geq 0, \quad a_{i j} \leq 0 \text { for } i \neq j, \quad a_{j}=\sum_{i \in I} a_{i j} \geq 0 .
$$

$A$ matrix with the compartmental structure with zero column sums is called conservative. If $A$ is compartmental then $B=A^{T}$ is called a matrix of positive type. A matrix of positive type $B$ is called conservative if $A=B^{T}$ is (compartmental) conservative matrix.

Hence, we consider a class of discretizations $A_{n}$ with the following properties:

$$
\begin{aligned}
& \left(A_{n}\right)_{\boldsymbol{k} \boldsymbol{k}}>0, \quad\left(A_{n}\right)_{\boldsymbol{k} \boldsymbol{l}} \leq 0, \quad \boldsymbol{l} \neq \boldsymbol{k}, \\
& \sum_{\boldsymbol{k}}\left(A_{n}\right)_{\boldsymbol{k} \boldsymbol{l}} \geq 0 .
\end{aligned}
$$

Additionally, there must exist a positive number $\sigma^{2}$ such that

$$
\sigma^{2}=\sup _{n} h(n)^{2} \sup \left\{\left(A_{n}\right)_{\boldsymbol{k} \boldsymbol{k}}: \boldsymbol{k} \in I_{n}\right\}
$$

Then $Q_{n}=\sigma^{2} I-h^{2} A_{n} \geq 0$ and $\left|Q_{n}\right|_{1} \leq \sigma^{2}$. This fact enables a decomposition of $A_{n}$ as $A_{n}=h^{-2}\left(\sigma^{2} I-Q_{n}\right)$. Hence, the resolvents

$$
R\left(\lambda, A_{n}\right)=\frac{h^{2}}{\sigma^{2}} \frac{1}{1+\lambda^{2} / \sigma^{2}} \sum_{k=0}^{\infty} \frac{1}{\left(1+\lambda^{2} / \sigma^{2}\right)^{k}} Q_{n}^{k}
$$

are non-negative matrices on $G_{n}, \mathbf{|} R\left(\lambda, A_{n}\right) \mathbf{|}_{1} \leq 1 / \lambda$.

Let $I^{\prime} \subset I$. Then $A^{\prime}=\left\{a_{i j}\right\}_{I^{\prime} I^{\prime}}$ is called a diagonal submatrix of $A=\left\{a_{i j}\right\}_{I I}$. A non-negative matrix $A=\left\{a_{i j}\right\}_{I I}$ is called irreducible if for each finite index set $I^{\prime} \subset I$ the corresponding diagonal submatrix is irreducible. A non-negative matrix which is not irreducible is called reducible. Thus a non-negative matrix $A$ is irreducible if for each finite subsets $I^{\prime} \subset I$ there exists $m \in \mathbb{N}$ such that the entries $p_{i j}$ of $P=A^{m}$ are positive for $i, j \in I^{\prime}$.

LEMMA 3.1 Let $A_{n}$ possess either the compartmental structure or be of positive type. Then the matrix $R\left(\lambda, A_{n}\right), \lambda>0$ on $G_{n}$ is positive iff it is irreducible.

Let the matrices $A_{n}$ on $G_{n}$ be discretizations of the differential operator (1) and let there be defined linear systems:

$$
A_{n} \mathbf{u}_{n}=\boldsymbol{\mu}_{n},
$$

where $\mathbf{u}_{n}, \boldsymbol{\mu}_{n} \in l\left(G_{n}(D)\right)$. We say that the system (9) numerically approximates the boundary value problem (4). The columns $\mathbf{u}_{n}$ are called grid-solutions. Obviously, $\boldsymbol{\mu}_{n}$ are discretizations of $\mu$. If the matrix $A_{n}$ is compartmental or of positive type we have $A_{n}=D_{n}-B_{n}$, where the diagonal matrix $D_{n}$ has positive entries and non-negative matrix $B_{n}$ has zero diagonal entries. Finite difference equations (9) can be rewritten as $\mathbf{u}_{n}=D_{n}^{-1} B_{n} \mathbf{u}_{n}+D_{n}^{-1} \boldsymbol{\mu}_{n}$ or componentwise as

$$
u_{\boldsymbol{k}}=\sum_{\boldsymbol{l} \neq \boldsymbol{k}}\left(D_{n}^{-1} B_{n}\right)_{\boldsymbol{k} \boldsymbol{l}} u_{\boldsymbol{l}}+d_{\boldsymbol{k} \boldsymbol{k}}^{-1} \boldsymbol{\mu}_{\boldsymbol{k}}
$$


Hence, if $A_{n}$ is compartmental or of positive type, then the obtained numerical scheme is monotone [BS].

For a matrix $A_{n}$ on $G_{n}$ we define numerical neighborhoods

$$
\mathcal{N}(\boldsymbol{x})=\left\{\boldsymbol{y} \in G_{n}: \boldsymbol{x}=h \boldsymbol{k}, \boldsymbol{y}=h \boldsymbol{l},\left(A_{n}\right)_{\boldsymbol{k} \boldsymbol{l}} \neq 0\right\} .
$$

Obviously, numerical neighborhoods of a system matrix and stencils of the corresponding finite difference schemes are mutually related.

\section{Imbedding of grid-functions into $W_{2}^{1}$-space}

Let us define the quadratic functional on $l\left(G_{n}\right)$ by $q(\mathbf{u})=\sum_{i}^{d}\left|U_{i} \mathbf{u}\right|_{2}^{2}$ and $q_{R}(\mathbf{u})=$ $\operatorname{vol}(R) \sum_{i}^{d}\left|U_{i}\left(r_{i}\right) \mathbf{u}\right|_{R 2}^{2}$ on $l\left(G_{n}(R)\right)$. It is understood that $q_{R}=q$ for $G_{n}(R)=G_{n}$. There exist symmetric matrices $Q_{n}$ on $G_{n}$ such that $q_{R}\left(\mathbf{u}_{n}\right)=\left\langle\mathbf{u} \mid Q_{n} \mathbf{u}\right\rangle$. Discrete analogs of $W_{2}^{1}$-spaces are spaces of those $\mathbf{u}_{n} \in l\left(G_{n}(R)\right)$ for which the norm $\left.|\cdot|\right|_{R 2,1}$ :

$$
|\mathbf{u}|_{R 2,1}^{2}=|\mathbf{u}|_{R 2}^{2}+q_{R}(\mathbf{u}),
$$

is finite. By convention $|\cdot|_{2,1}=\left.\mathbf{I} \cdot\right|_{R 2,1}$ for $r_{i}=1$. The subspace of gridfunctions $\mathbf{u} \in w_{2}^{1}\left(G_{n}(R)\right)$ for which $\mathbf{u}_{n}=\mathbb{1}_{G_{n}(D)} \mathbf{u}_{n}$ is denoted by $w_{2}^{1}\left(G_{n}(R, D)\right)$. Hence, $w_{2}^{1}\left(G_{n}(R, D)\right)$ for $\boldsymbol{r}=\mathbf{1}$ is denoted by $w_{2}^{1}\left(G_{n}(D)\right)$. The restriction of $q_{R}$ on $G_{n}(R, D)$ is represented as $q_{R}(\mathbf{u})=\left\langle\mathbf{u} \mid Q_{n}(D) \mathbf{u}\right\rangle$, where $Q_{n}(D)$ is a symmetric matrix on $G_{n}(R, D)$. By using the fact that the negative Laplacean on $\dot{C}^{(2)}(\bar{D})$ and its discretizations $-\sum_{i} V_{i} U_{i}$ in $l_{2}\left(G_{n}(D)\right)$ have positive minimal eigenvalues, we can derive the following result.

LEMMA 3.2 Let $D$ be bounded and $Q_{n}(D)$ be irreducible on $G_{n}(R, D)$. Then the norms (10) and $q_{R}(\cdot)^{1 / 2}$ are equivalent in $w_{2}^{1}\left(G_{n}(R, D)\right)$,

$$
q_{R}(\cdot)^{1 / 2} \geq\left.\beta|\cdot|\right|_{R 2,1},
$$

where $\beta$ is independent of $n$.

Let us consider a norm $|\cdot|_{R 2,1}$ on $l\left(G_{n}(R)\right)$ defined by (10). Any such norm is a semi-norm on $l\left(G_{n}\right)$. Our object of interest are quadratic functionals:

$$
\begin{aligned}
& |\mathbf{u}|_{a v g, 2,1}^{2}=\frac{1}{\operatorname{vol}(R)} \sum_{R}|\mathbf{u}|_{R 2,1}^{2} \\
& =|\mathbf{u}|_{2}^{2}+\frac{1}{\operatorname{vol}(R)} \sum_{i=1}^{d}\left|U_{i}\left(r_{i}\right) \mathbf{u}\right|_{R 2}^{2} \leq|\mathbf{u}|_{R 2,1}^{2} . \quad \mathbf{u} \in l\left(G_{n}\right) \text {. }
\end{aligned}
$$

Then $|\cdot|_{\text {avg,2,1 }}$ is a norm on $l\left(G_{n}\right)$. Unfortunately, it is not equivalent to $\left.|\cdot|\right|_{2,1}$ uniformly with respect to $n$.

An element (column) $\mathbf{u}_{n} \in l\left(G_{n}\right)$ can be associated to a continuous function on $\mathbb{R}^{d}$ in various ways. Here is utilized a mapping $l\left(G_{n}\right) \mapsto C\left(\mathbb{R}^{d}\right)$ which is defined in terms of hat functions. Let $\chi$ be the canonical hat function on $\mathbb{R}$, centered at the origin and having the support $[-1,1]$. Then $z \mapsto \phi(h, x, z)=\chi\left(h^{-1}(z-h x)\right)$ is the hat function on $\mathbb{R}$, centered at $x \in \mathbb{R}$ with support $[x-h, x+h]$. The functions $\boldsymbol{z} \mapsto \phi_{\boldsymbol{k}}(\boldsymbol{z})=\prod_{i=1}^{d} \phi\left(h, x_{i}, z_{i}\right), x_{i}=h k_{i}$, define $d$-dimensional hat functions with supports $S(\mathbf{1}, \boldsymbol{x})=\prod_{i}\left[x_{i}-h, x_{i}+h\right]$. The functions $\phi_{\boldsymbol{k}}(\cdot) \in G_{n}$, span a linear space, denoted by $E_{n}\left(\mathbb{R}^{d}\right)$. Let $\mathbf{u}_{n} \in l\left(G_{n}\right)$ have the entries $u_{n \boldsymbol{k}}=\left(\mathbf{u}_{n}\right)_{\boldsymbol{k}}$. Then the function $u(n)=\sum_{\boldsymbol{k} \in \mathbb{Z}^{d}} u_{n \boldsymbol{k}} \phi_{\boldsymbol{k}}$ belongs to $E_{n}\left(\mathbb{R}^{d}\right)$ and defines imbedding of grid-functions into the space of continuous functions. We denote the corresponding mapping by $\Phi_{n}: l\left(G_{n}\right) \mapsto E_{n}\left(\mathbb{R}^{d}\right)$. Obviously there exists $\Phi_{n}^{-1}: E_{n}\left(\mathbb{R}^{d}\right) \mapsto l\left(G_{n}\right)$ and the spaces $l\left(G_{n}\right)$ and $E_{n}\left(\mathbb{R}^{d}\right)$ are isomorphic with respect to the pair of mappings $\Phi_{n}, \Phi_{n}^{-1}$. It is clear that $E_{n}\left(\mathbb{R}^{d}\right) \subset E_{n+1}\left(\mathbb{R}^{d}\right)$ and the space of functions $\cup_{n} E_{n}\left(\mathbb{R}^{d}\right)$ is dense in $L_{p}\left(\mathbb{R}^{d}\right), p \in[1, \infty)$, as well as in $\dot{C}\left(\mathbb{R}^{d}\right)$. Let us mention that $\sum_{\boldsymbol{k}} \phi_{\boldsymbol{k}}=1$ on $\mathbb{R}^{d}$. 
Now we consider another collection of basis functions. To each $\boldsymbol{x}=h \boldsymbol{k} \in G_{n}(R)$ there is associated a $d$-dimensional hat function

$$
\psi_{\boldsymbol{k}}(\boldsymbol{x})=\prod_{i=1}^{d} \chi\left(\frac{x_{i}-h k_{i}}{h r_{i}}\right),
$$

obviously, with the support $S(\boldsymbol{r}, \boldsymbol{x})=\prod_{i}\left[x_{i}-r_{i} h, x_{i}+r_{i} h\right]$. They span a linear space denoted by $E_{n}\left(R, \mathbb{R}^{d}\right)$. Again we have $\sum_{\boldsymbol{k}} \psi_{\boldsymbol{k}}=1$ on $\mathbb{R}^{d}$. The mappings $\Phi_{n}, \Phi_{n}^{-1}$ cannot be applied to elements of $l\left(G_{n}(R)\right)$ and $E_{n}\left(R, \mathbb{R}^{d}\right)$, respectively. Therefore we define restrictions $\Phi_{n}(R): l\left(G_{n}(R)\right) \rightarrow E_{n}\left(R, \mathbb{R}^{d}\right)$ and $\Phi_{n}^{-1}(R)$ by the following expression:

$$
u(n)=\Phi_{n}(R) \mathbf{u}_{n}=\sum_{\boldsymbol{k}}\left(\mathbf{u}_{n}\right)_{\boldsymbol{k}} \psi_{\boldsymbol{k}} .
$$

If we have to emphasize that $u(n)$ is related to a particular set of parameters $R$, then we use an extended denotation $u(R, n)$.

The following results are necessary in our proof of the consistency in Section 5

LEMMA 3.3 Let for each $n \in \mathbb{N}$ there exist columns $\mathbf{u}_{n}, \mathbf{v}_{n} \in l\left(G_{n}(R)\right)$ such that $h^{d}\left|\mathbf{u}_{n}\right|_{R 2}^{2} \leq c, h^{d}\left|\mathbf{v}_{n}\right|_{R 2}^{2} \leq c$, where $c>0$ is independent of $n$. Let $u(n)=\Phi_{n}(R) \mathbf{u}_{n}$ and $v(n)=\Phi_{n}(R) \mathbf{v}_{n}$. If

$$
\lim _{n} h^{d} \sup _{|\boldsymbol{k}-\boldsymbol{l}|=|\boldsymbol{r}|}\left|\left(Z_{n}(\boldsymbol{k}-\boldsymbol{l})-I\right) \mathbf{u}_{n}\right|_{R 2}^{2}=0,
$$

then

$$
\lim _{n}\left((v(n) \mid u(n))-h^{d}\left(\mathbf{v}_{n} \mid \mathbf{u}_{n}\right)_{R}\right)=0 .
$$

It is easy to check $\|u(n)\|_{2}^{2} \leq h^{d}\left|\mathbf{u}_{n}\right|_{2}^{2}$. There holds a similar inequality for any pair $\partial_{i} u, U_{i} \mathbf{u}_{n}$. First one verifies

$$
\left(\partial_{i} v(n) \mid \partial_{i} u(n)\right)=\left(\mathrm{\Xi}_{i}\left(r_{i} h\right) v(n) \mid \mathrm{\Xi}_{i}\left(h r_{i}\right)(u(n))=\left(U_{i}\left(r_{i}\right) \mathbf{v}_{n} \mid U_{i}\left(r_{i}\right) \mathbf{u}_{n}\right),\right.
$$

so that the following Theorem holds.

THEOREM 3.1 Let sequences of functions $v(n), u(n), n \in \mathbb{N}$, be defined by (12). Then

$$
\begin{gathered}
\left|\sum_{i=1}^{d}\left(\partial_{i} v(n) \mid \partial_{i} u(n)\right)\right| \leq h^{d} q_{R}\left(\mathbf{v}_{n}\right)^{1 / 2} q_{R}\left(\mathbf{u}_{n}\right)^{1 / 2} . \\
\leq h^{d} \min \left\{\begin{array}{l}
\mid U_{i}\left(r_{i}\right) \mathbf{v} \mathbf{|}_{2} \sup \left\{\left.\mathbf{|}(Z(w, j)-I) U_{j}\left(r_{j}\right) \mathbf{u}\right|_{2}:|w| \leq r_{j} h\right\} \\
\left|U_{j}\left(r_{j}\right) \mathbf{u}\right|_{2} \sup \left\{\left|(Z(w, i)-I) U_{i}\left(r_{i}\right) \mathbf{v}\right|_{2}:|w| \leq r_{i} h\right\}
\end{array}\right.
\end{gathered}
$$

Now we can get the following useful result involving the norm $\|u(n)\|_{2,1}$ and its averaged value:

$$
\|u(n)\|_{a v g, 2,1}^{2}=\frac{1}{\operatorname{vol}(R)} \sum_{R}\|u(R, n)\|_{2,1}^{2} .
$$

COROLLARY 3.1 Let $u(n)=\Phi_{n} \mathbf{u}_{n}$. There exists $\sigma^{2} \in(0,1)$, independent of $n$, such that

$$
\begin{aligned}
& \left(1-\sigma^{2}\right) h^{d}\left|\mathbf{u}_{n}\right|_{R 2,1}^{2} \leq\|u(R, n)\|_{2,1}^{2} \leq h^{d}\left|\mathbf{u}_{n}\right|_{R 2,1}^{2} \\
& \left(1-\sigma^{2}\right) h^{d}\left|\mathbf{u}_{n}\right|_{\text {avg,2,1 }}^{2} \leq\|u(n)\|_{\text {avg }, 2,1}^{2} \leq h^{d}\left|\mathbf{u}_{n}\right|_{\text {avg, }, 2,1}^{2}
\end{aligned}
$$


An element $u \in W_{2}^{1}\left(\mathbb{R}^{d}\right)$ does not belong necessary to $E_{n}\left(R, \mathbb{R}^{d}\right)$. In order to approximate $u$ with elements of $E_{n}\left(R, \mathbb{R}^{d}\right)$ we define:

$$
\hat{u}(n)=\sum_{\boldsymbol{k} \in I_{n}(R)}\left\|\psi_{\boldsymbol{k}}\right\|_{1}^{-1}\left(\psi_{\boldsymbol{k}} \mid u\right) \psi_{\boldsymbol{k}}
$$

The numbers $\left\|\psi_{\boldsymbol{k}}\right\|_{1}^{-1}\left(\psi_{\boldsymbol{k}} \mid u\right)$ are called Fourier coefficients of $u$.

The basic result for our proof of convergence of approximate solutions is formulated by using the quantity $\Gamma_{p}(\boldsymbol{w}, u)$ defined by:

$$
\Gamma_{p}(\boldsymbol{w}, u)=\|(Z(\boldsymbol{w})-I) u\|_{p} .
$$

The kernels

$$
\omega_{n}(\boldsymbol{x}, \boldsymbol{y})=\sum_{\boldsymbol{k}} \frac{1}{\left\|\psi_{\boldsymbol{k}}\right\|_{1}} \psi_{\boldsymbol{k}}(\boldsymbol{x}) \psi_{\boldsymbol{k}}(\boldsymbol{y})
$$

define an integral operator which is denoted by $K_{n}$. Actually, the kernels $\omega_{n}$ define a $\delta$-sequence of functions on $\mathbb{R}^{d} \times \mathbb{R}^{d}$ and $K_{n}$ converge strongly in $L_{p^{-}}$-spaces to unity:

COROLLARY 3.2 Let $p \in[1, \infty]$. Then

(i) $\left\|K_{n}\right\|_{p} \leq 1$.

(ii) There is a positive number $\kappa(R)$, independent of $n$, such that $\left\|\left(I-K_{n}\right) u\right\|_{p} \leq$ $\kappa(R) \sup \left\{\Gamma_{p}(\boldsymbol{w}, u):\left|w_{i}\right| \leq h r_{i}\right\}$.

(iii) The operator $K_{n} \in L\left(L_{2}\left(\mathbb{R}^{d}\right), L_{2}\left(\mathbb{R}^{d}\right)\right)$ has the spectrum equal $[0,1]$.

If necessary, we write $K_{n}(R)$ instead of $K_{n}$ to emphasize that the integral operator is defined with a basis $\psi_{\boldsymbol{k}}$ in $E_{n}\left(R, \mathbb{R}^{d}\right)$ for a particular value of parameter $R$.

THEOREM 3.2 Let $v, u \in W_{2}^{1}\left(\mathbb{R}^{d}\right)$ and $\hat{u}(n), \hat{v}(n)$ be defined by 14). Then

$$
\begin{gathered}
|(\hat{v}(n) \mid \hat{u}(n))-(v \mid u)| \leq c(R) \min \left\{\begin{array}{l}
\|u\|_{2} \sup _{|\boldsymbol{w}|=h} \Gamma_{2}(\boldsymbol{w}, v), \\
\|v\|_{2} \sup |\boldsymbol{w}|=h
\end{array} \Gamma_{2}(\boldsymbol{w}, u),\right. \\
|(\partial \hat{v}(n) \mid \partial \hat{u}(n))-(\partial v \mid \partial u)| \leq c(R) \min \left\{\begin{array}{l}
\|\partial v\|_{2}\left[\|\partial u-\exists u\|_{2}+\sup _{|\boldsymbol{w}|=h} \Gamma_{2}(\boldsymbol{w}, \partial u)\right], \\
\|\partial u\|_{2}\left[\|\partial v-\exists v\|_{2}+\sup _{|\boldsymbol{w}|=h} \Gamma_{2}(\boldsymbol{w}, \partial v)\right],
\end{array}\right.
\end{gathered}
$$

where $c(R)$ is n-independent.

The procedure by which the norm (11) is accompanied with the norms (10) is thoroughly utilized throughout this work. Therefore we define the procedure generally. Let a collection of functions $u(R, n)$ defined by (12) or grid-functions $\mathbf{u}_{n}(R)$ on $G_{n}(R)$ be denoted by $\mathfrak{C}(R)$. For a functional $F$ on $\mathfrak{C}(R)$ or $\mathfrak{C}(R) \times \mathfrak{C}(R)$ the averaged value $F_{\text {avg }}$ is defined as the arithmetic mean of functionals over all $\mathfrak{C}(R)$ or $\mathfrak{C}(R) \times \mathfrak{C}(R)$, respectively. For instance, the $W_{2}^{1}$-norm on $E_{n}\left(R, \mathbb{R}^{d}\right)$ has its averaged version denoted by $\|\cdot\|_{\text {avg }, 2,1}$ and explicitly expressed by (13). Another important example is a bilinear form $a(\cdot, \cdot)$ on $E_{n}\left(R, \mathbb{R}^{d}\right) \times E_{n}\left(R, \mathbb{R}^{d}\right)$. Its averaged version is defined by:

$$
a_{\text {avg }}(\hat{v}(n), \hat{u}(n))=\frac{1}{\operatorname{vol}(R)} \sum_{R} a(\hat{v}(R, n), \hat{u}(R, n)) .
$$

\section{Discretizations of elliptic operators}

Most of constructions and descriptions in this section are given for the differential operator $A_{0}$. At the end of section we briefly explain how to include discretizations for the lower order terms in (1). 


\subsection{Constant diffusion tensor}

Schemes and some of results regarding the convergence can be easily described for the case of classical second order elliptic operator $A_{0}=-\sum_{i j} a_{i j} \partial_{i} \partial_{j}$, where the functions $a_{i j}$ are Hölder continuous on $\mathbb{R}^{d}$. At the beginning we analyze the case of constant diffusion tensor $\left\{a_{i j}\right\}_{11}^{d d}$.

A standard approach to a generation of discretizations of differential operators is based on utilization of finite difference operators approximating $\partial_{i}, \partial_{i} \partial_{j}$. So, by using the forward and backward difference operators $\Xi_{i}\left(p_{i} h_{i}\right), \widehat{\vec{a}}_{i}\left(p_{i} h_{i}\right)$ we can define various discretizations $\vec{a}_{i i} f(\boldsymbol{x})$ of $\partial_{i} \partial_{j} f$. In this way, the quadratic operator $-a_{i i}\left(\partial_{i}\right)^{2}-$ $a_{j j}\left(\partial_{j}\right)^{2}$ is approximated by the standard central difference scheme $a_{i i} \Xi_{i i}+a_{j j} a_{j j}$ with positive diagonal entries and non-positive off-diagonal entries. For $i \neq j$ finite differences are defined by:

$$
\frac{1}{h_{1} h_{2}}\left\{\begin{array}{c}
\partial_{i} \partial_{j} f(\boldsymbol{x}) \rightarrow \Xi_{i j} f(\boldsymbol{x})= \\
f\left(\boldsymbol{x} \pm \boldsymbol{e}_{i} h_{i} \pm \boldsymbol{e}_{j} h_{j}\right)-f\left(\boldsymbol{x} \pm \boldsymbol{e}_{i} h_{i}\right)-f\left(\boldsymbol{x} \pm \boldsymbol{e}_{j} h_{j}\right)+f(\boldsymbol{x}), \\
-f\left(\boldsymbol{x} \pm \boldsymbol{e}_{i} h_{i} \mp \boldsymbol{e}_{j} h_{j}\right)+f\left(\boldsymbol{x} \pm \boldsymbol{e}_{i} h_{i}\right)+f\left(\boldsymbol{x} \mp \boldsymbol{e}_{j} h_{j}\right)-f(\boldsymbol{x}) .
\end{array}\right.
$$

If $a_{i j} \geq 0$, then $a_{i j} \partial_{i} \partial_{j}$ is approximated by the half sum of the first two possibilities, otherwise by the half sum of the second two possibilities. Hence, for the $d$-dimensional case

$$
\begin{aligned}
& A_{\boldsymbol{k} \boldsymbol{k} \pm r_{i} \boldsymbol{e}_{i}}=-\frac{1}{h^{2} r_{i}}\left[\frac{1}{r_{i}} a_{i i}-\sum_{m \neq i} \frac{1}{r_{m}}\left|a_{m i}\right|\right],
\end{aligned}
$$

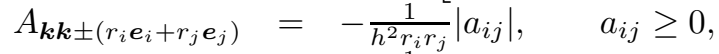

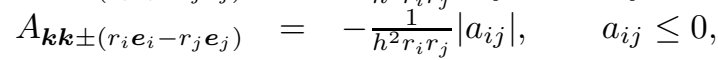

while the diagonal entries are calculated as the negative sum of off-diagonal ones. To a given diffusion tensor $a=\left\{a_{i j}\right\}_{11}^{d d}$ we associate an auxiliary tensor $\hat{a}$ defined by:

$$
\hat{a}_{i i}=a_{i i}, \quad \hat{a}_{i j}=-\left|a_{i j}\right| \quad i \neq j .
$$

By using Perron-Frobenius theorem one can easily prove the following assertion. There exist grid-steps $h$ such that the matrix $A_{n}$ defined by (16) has the compartmental structure iff the auxiliary diffusion tensor $\hat{a}$ is positive definite.

In case of $d=2$ the tensors $a, \hat{a}$ are simultaneously positive definite or not. For higher dimensions $\hat{a}$ can be indefinite although $a$ is positive definite.

Apart from this standard approach to discretizations of differential operators there can be used constructions avoiding finite difference operators. One of possibilities is based on the following simple result:

DEFINITION 4.1 A matrix $A_{n}$ with a finite band is called a discretization of the differential operator $A_{0}=-\sum_{i j} a_{i j} \partial_{i} \partial_{j}$ if the equalities

$$
(A u)(\boldsymbol{k})=\sum_{\boldsymbol{l}}\left(A_{n}\right)_{\boldsymbol{k} \boldsymbol{l}} u_{\boldsymbol{l}}
$$

are valid for any polynomial $\boldsymbol{x} \rightarrow u(\boldsymbol{x})$ of the second degree.

LEMMA 4.1 Let $A_{n}$ be discretizations of the differential operator $A_{0}$ and $\mathbf{u}_{n}=$ $R\left(\lambda, A_{n}\right) \boldsymbol{\mu}_{n}$ such that $\left|R\left(\lambda, A_{n}\right)\right| \infty$ is bounded uniformly with respect to $n \in \mathbb{N}$. Then the sequence of functions $\mathfrak{U}=\{u(n): n \in \mathbb{N}\} \subset \cup_{n} E_{n}(D)$ converges in $\dot{C}^{(\alpha)}\left(\mathbb{R}^{d}\right)$ to the solution $u=R\left(\lambda, A_{0}\right) f$ of (4).

PRoOF: One can utilize arguments in standard proofs of the factor convergence of grid solutions as described in [RM]. In order to prove the convergence in $C^{(\alpha)}(\bar{D})$ it is necessary to have the condition (17) as demonstrated in [LR1].

QED 
A useful application of this lemma is the discretization defined by:

$$
\begin{aligned}
& A_{\boldsymbol{k} \boldsymbol{k} \pm \boldsymbol{e}_{i}} \quad=-\frac{1}{h^{2}}\left[a_{i i}-\sum_{m \neq i} \frac{r_{i}}{r_{m}}\left|a_{m i}\right|\right], \\
& A_{\boldsymbol{k} \boldsymbol{k} \pm\left(r_{i} \boldsymbol{e}_{i}+r_{j} \boldsymbol{e}_{j}\right)}=-\frac{1}{h^{2} r_{i} r_{j}}\left|a_{i j}\right|, \quad a_{i j} \geq 0,
\end{aligned}
$$

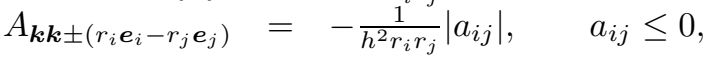

where $r_{i}, r_{j} \in \mathbb{N}$. Of course, a convex combination of the system matrices (18) is again a system matrix discretizing the classical differential operator $A_{0}=-\sum_{i j} a_{i j} \partial_{i} \partial_{j}$.

In the case of two dimensions the obtained structures of system matrix can be classified into two groups by using the corresponding numerical neighborhoods. The grid $G_{n}=\left\{h\left(k \boldsymbol{e}_{1}+l \boldsymbol{e}_{2}\right): k, l \in \mathbb{Z}\right\}$ has the corresponding index set of indices $\boldsymbol{k}=(k, l)$. Possible numerical neighborhoods $\mathcal{N}(\boldsymbol{x})$ for the respective methods (16), (18), are illustrated in Figure 4.1.

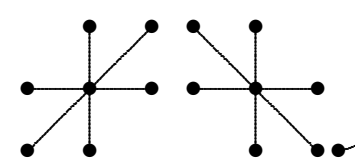

(a): $a_{12} \geq 0,(\mathrm{~b}): a_{12} \leq 0$

$$
r_{1}=r_{2}=1
$$

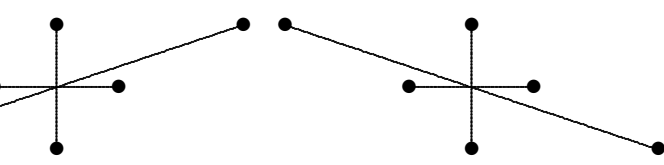

(c): $a_{12} \geq 0$

(d): $a_{12} \leq 0$

Figure 4.1

In order to discretize (44) by monotone schemes we must have discretizations $A_{n}$ of (11) in terms of matrices of positive type. If we utilize discretizations (18) the following inequalities must be satisfied:

$$
\sum_{m \neq i} \frac{1}{h r_{m}}\left|a_{m i}\right| \leq \frac{1}{h r_{i}} a_{i i}, \quad i \in\{1,2, \ldots, d\},
$$

and the following Lemma follows easily.

LEMMA 4.2 There exist natural numbers $r_{i}$ such that the matrix $A_{n}$ defined by (18) is of positive type iff the auxiliary diffusion tensor $\hat{a}$ is positive definite.

As already mentioned, in higher dimensions $\hat{a}$ can be indefinite although $a$ is positive definite. In such cases the simple schemes (18) cannot give us matrices of positive type. Rotations of coordinates are necessary to get system matrices of positive type. Naturally, so obtained schemes are also covered by Definition 4.1

All the results of this subsection can be straightforwardly extended to cases with a general tensor-valued function $\boldsymbol{x} \rightarrow a(\boldsymbol{x})$. Numbers $a_{i j}$ in Expressions (16), (18) have to be replaced with the values $a_{i j}(\boldsymbol{x})$ at the considered grid-knot $\boldsymbol{x} \in G_{n}$. The condition of Lemma 4.2 must be satisfied uniformly with respect to $\boldsymbol{x} \in G_{n}$.

\subsection{Operators in divergence form}

Discretizations of $A_{0}(\boldsymbol{x})$ which are analyzed here are constructed by using several rules. Rules can be described vaguely as follows. The set $\mathbb{R}^{d}$ is discretized by $G_{n}(A) \subset G_{n}, u$ by $\mathbf{u}_{n}, \partial_{i} u$ by $\left(U_{i}\left(p_{i}\right) \mathbf{u}_{n}\right)$, where $p_{i}$ are generally $\boldsymbol{x}$-dependent, $a_{i j}$ are discretized by $a_{i j}\left(\boldsymbol{x}_{i j}(n, \boldsymbol{x})\right), \boldsymbol{x} \in G_{n}(A)$, where $\boldsymbol{x}_{i j}(n, \cdot): \mathbb{R}^{d} \mapsto \mathbb{R}^{d}$ are certain functions, and integral is replaced by the corresponding Riemmanian sum of factors calculated at grid-knots. Thus the form (44) is discretized by a sequence of forms $\mathbf{v}_{n}, \mathbf{u}_{n} \mapsto h(n)^{d}\left\langle\mathbf{v}_{n} \mid A_{n} \mathbf{u}_{n}\right\rangle$. Without loss of generality, we may assume that entries of resulting matrices $A_{n}$ are defined by functions $\boldsymbol{x}_{i j}(n, \cdot): \mathbb{R}^{d} \mapsto \mathbb{R}^{d}$ and values $a_{i j}\left(\boldsymbol{x}_{i j}(n, \boldsymbol{z})\right), \boldsymbol{z} \in G_{n}$. 


\section{Standard schemes in two dimension}

We consider a subset of grid-knots $G_{n}(R)=\boldsymbol{r}_{0}+\left\{k h r_{1} \boldsymbol{e}_{1}+l h r_{2} \boldsymbol{e}_{2}: k, l \in \mathbb{Z}\right\} \subset G_{n}$ and the forms

$$
a_{n}(v, u)=\sum_{i j=1}^{2} \sum_{\boldsymbol{x} \in G_{n}(R)}\left(\Xi_{i}\left(h r_{i}\right) v\right)(\boldsymbol{x}) a_{i j}\left(\boldsymbol{x}+\frac{h}{2}\left(r_{1} \boldsymbol{e}_{1}+r_{2} \boldsymbol{e}_{2}\right)\right)\left(\Xi_{j}\left(h r_{j}\right) u\right)(\boldsymbol{x}) .
$$

The non-trivial off-diagonal matrix entries of $A_{n}$ are defined as follows:

$$
\begin{aligned}
& \left(A_{n}\right)_{\boldsymbol{k} \boldsymbol{k}_{1} \boldsymbol{e}_{1}}=-\frac{1}{h^{2} r_{1}}\left(\frac{1}{r_{1}} a_{11}^{ \pm+}-\frac{1}{r_{2}}\left|a_{12}^{ \pm+}\right|\right), \\
& \left(A_{n}\right)_{\boldsymbol{k} \boldsymbol{k} \pm r_{2} \boldsymbol{e}_{2}}=-\frac{1}{h^{2} r_{2}}\left(\frac{1}{r_{2}} a_{22}^{+ \pm}-\frac{1}{r_{1}}\left|a_{12}^{+ \pm}\right|\right), \\
& \left(A_{n}\right)_{\boldsymbol{k} \boldsymbol{k} \pm\left(r_{1} e_{1}-r_{2} e_{2}\right)}=-\frac{1}{h^{2} r_{1} r_{2}}\left|a_{12}^{ \pm \mp}\right|
\end{aligned}
$$

and

$$
a_{i j}^{ \pm+}=a_{i j}\left(\boldsymbol{x}+\frac{h}{2}\left( \pm r_{1} \boldsymbol{e}_{1}+r_{2} \boldsymbol{e}_{2}\right)\right), \quad a_{i j}^{ \pm-}=a_{i j}\left(\boldsymbol{x}+\frac{h}{2}\left( \pm r_{1} \boldsymbol{e}_{1}-r_{2} \boldsymbol{e}_{2}\right)\right) .
$$

If $a_{12} \leq 0$ and brackets in (19) have positive values, then the scheme (19) defines a matrix $A_{n}$ with the compartmental structure; this scheme is called the first scheme. Numerical neighborhoods are defined by $\mathcal{N}^{(-)}(\boldsymbol{x})=\boldsymbol{x}+\mathcal{N}^{(-)}(\mathbf{0})$, where

$$
\mathcal{N}^{(-)}(\mathbf{0})=\{\mathbf{0}\} \cup\left\{ \pm h r_{1} \boldsymbol{e}_{1} \pm h r_{2} \boldsymbol{e}_{2}, \quad \pm\left(h r_{1} \boldsymbol{e}_{1}-h r_{2} \boldsymbol{e}_{2}\right)\right\} .
$$

An illustration of a set $\mathcal{N}^{(-)}(\mathbf{0})$ is given in Figure 4.1(b).

For the second scheme the grid $G_{n}(R)$ is defined as before while

$$
\begin{aligned}
a_{n}(v, u) & =\sum_{i j=1}^{2} \sum_{\boldsymbol{x} \in G_{n}(R)}\left(\Xi_{i}\left((-)^{i-1} h r_{i}\right) v\right)(\boldsymbol{x}) \\
& \times a_{i j}\left(\boldsymbol{x}+\frac{1}{2}\left(h r_{1} \boldsymbol{e}_{1}-h r_{2} \boldsymbol{e}_{2}\right)\right)\left(\Xi_{j}\left((-)^{j-1} h r_{j}\right) u\right)(\boldsymbol{x}) .
\end{aligned}
$$

Off-diagonal matrix entries are:

$$
\begin{aligned}
& \left(A_{n}\right)_{\boldsymbol{k} \boldsymbol{k} \pm r_{1} e_{1}}=-\frac{1}{h^{2} r_{1}}\left(\frac{1}{r_{1}} a_{11}^{ \pm-}-\frac{1}{r_{2}}\left|a_{12}^{ \pm-}\right|\right) \\
& \left(A_{n}\right)_{\boldsymbol{k} \boldsymbol{k} \pm r_{2} e_{2}}=-\frac{1}{h^{2} r_{2}}\left(\frac{1}{r_{2}} a_{22}^{+ \pm}-\frac{1}{r_{1}}\left|a_{12}^{+ \pm}\right|\right) \\
& \left(A_{n}\right)_{\boldsymbol{k} \boldsymbol{k} \pm\left(r_{1} e_{1}+r_{2} e_{2}\right)}=-\frac{1}{h^{2} r_{1} r_{2}} a_{12}^{ \pm \pm} .
\end{aligned}
$$

If $a_{12} \geq 0$ and brackets in (20) have positive values the compartmental structure is again attained. The set $G_{n}(R)$ is unchanged while the numerical neighborhoods have different structure, $\mathcal{N}^{(+)}(\boldsymbol{x})=\boldsymbol{x}+\mathcal{N}^{(+)}(\mathbf{0})$, where now

$$
\mathcal{N}^{(+)}(\mathbf{0})=\{\mathbf{0}\} \cup\left\{ \pm h r_{1} \boldsymbol{e}_{1} \pm h r_{2} \boldsymbol{e}_{2}, \quad \pm\left(h r_{1} \boldsymbol{e}_{1}+h r_{2} \boldsymbol{e}_{2}\right)\right\},
$$

as illustrated in Figure 4.1(a).

For each scheme there exists only one $\boldsymbol{x}^{(\mp)}(n, \cdot)$ (instead of 3 mappings $\boldsymbol{x}_{i j}(n, \cdot)$ ) which is implicitly defined by expressions of $a_{i j}^{\mp \mp}$. Thus, for the first and second schemes we have the following respective expressions:

$$
\begin{aligned}
& \boldsymbol{x}^{(-)}(n, \boldsymbol{x}, \boldsymbol{r})=\boldsymbol{x}+h 2^{-1}\left(r_{1} \boldsymbol{e}_{1}+r_{2} \boldsymbol{e}_{2}\right), \\
& \boldsymbol{x}^{(+)}(n, \boldsymbol{x}, \boldsymbol{r})=\boldsymbol{x}+h 2^{-1}\left(r_{1} \boldsymbol{e}_{1}-r_{2} \boldsymbol{e}_{2}\right)
\end{aligned}
$$

where $\boldsymbol{x}^{(\mp)}$ contain explicitly the parameter $\boldsymbol{r}$. 


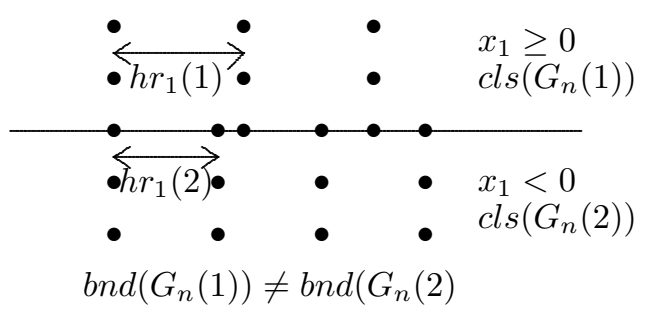

Figure 4.2
In a general two-dimensional case the function $a_{12}$ may vary and the compartmental structure of $A_{n}$ can be broken at some grid-knots. Besides, the function $a_{12}$ changes sign on $\mathbb{R}^{2}$ and the compartmental structure cannot be realized by using only one scheme for all the grid-knots. In the case of continuous diffusion tensor one can prove the following result. There exists a partition $\mathbb{R}^{d}=$ $\cup_{\mp} \cup_{l} D_{l}^{(\mp)}$ such that the function $a_{12}$ does not change sign on $D_{l}^{(\mp)}$. To each $D_{l}^{(\mp)}$ there correspond grid-steps $h r_{i}^{(\mp)}(l)$ such that the sets $G_{n}^{(\mp)}(l)=D_{l}^{(\mp)} \cap G_{n}$ are subgrids and $G_{n}(R)=\cup_{\mp l} G_{n}^{(\mp)}(l)$. To each grid-knot in $\operatorname{int}\left(G_{n}^{(\mp)}(l)\right)$ we associate one of neighborhoods $\mathcal{N}^{(\mp)}(\boldsymbol{x})$ and entries of $A_{n}$ defined by (19) or (20). Construction of entries of $A_{n}$ at grid-knots on the boundary of sets $D_{l}^{(\mp)}$ can bear a considerable amount of difficulties. For instance, one of simple cases is given by $G_{n}=G_{n}(1) \cup G_{n}(2)$ as illustrated in Figure 4.2, where we are faced with difficulties regarding the constructions of sets $\mathcal{N}(\boldsymbol{x}), \boldsymbol{x} \in\left\{\mathbb{R}^{2}: x_{2}=0\right\}$ and the corresponding entries of $A_{n}$.

\section{Extended schemes in two dimensions}

The mappings $\boldsymbol{x}^{(\mp)}(n, \boldsymbol{x}, \boldsymbol{r})$ in $\mathbb{R}^{2}$ are defined by (21), where the superscripts $( \pm)$ are related to the sign of $a_{12}$, i.e. to the cases $a_{12} \geq 0$ and $a_{12} \leq 0$, respectively. The bilinear form on $G_{n} \times G_{n}$

$$
\begin{gathered}
a_{n}^{(-)}(v, u)=\sum_{\boldsymbol{x} \in G_{n}}\left(\left[\sum_{i=1}^{2} a_{i i}\left(\boldsymbol{x}^{(-)}(n, \boldsymbol{x})\right)\left(\mathrm{\Xi}_{i}(h) v\right)(\boldsymbol{x})\left(\mathrm{\Xi}_{i}(h) u\right)(\boldsymbol{x})\right.\right. \\
\left.+\sum_{i \neq j} a_{i j}\left(\boldsymbol{x}^{(-)}(n, \boldsymbol{x}, \boldsymbol{r})\right)\left(\mathrm{\Xi}_{i}\left(r_{i} h\right) v\right)(\boldsymbol{x})\left(\mathrm{\Xi}_{j}\left(r_{j} h\right) u\right)(\boldsymbol{x})\right] \\
\left.+\sum_{i \neq j} a_{i j}\left(\boldsymbol{x}^{(-)}(n, \boldsymbol{x}, \boldsymbol{r})\right) \frac{r_{i}}{r_{j}}\left[\left(\mathrm{\Xi}_{i}(h) v\right)(\boldsymbol{x})\left(\mathrm{\Xi}_{i}(h) u\right)(\boldsymbol{x})-\left(\mathrm{\Xi}_{i}\left(r_{i} h\right) v\right)(\boldsymbol{x})\left(\mathrm{\Xi}_{i}\left(r_{i} h\right) u\right)(\boldsymbol{x})\right]\right)
\end{gathered}
$$

can be written as

$$
a_{n}^{(-)}(v, u)=\left\langle\mathbf{v} \mid A_{n} \mathbf{u}\right\rangle
$$

where $\boldsymbol{r} \in \mathbb{N}^{2}, \boldsymbol{x}^{(\mp)}(n, \boldsymbol{x})=\boldsymbol{x}^{(\mp)}(n, \boldsymbol{x}, \mathbf{1})$ by convention, $\mathbf{v}, \mathbf{u}$ are columns with entries $v(\boldsymbol{x}), u(\boldsymbol{x})$, and $A_{n}$ is the resulting system matrix. By utilizing the standard variational calculus we can derive entries of $A_{n}$. In order to rewrite the entries of $A_{n}$ in a concise form we use the following abbreviations:

$$
\begin{aligned}
& a_{i j}^{ \pm+}(\boldsymbol{r})=a_{i j}\left(\boldsymbol{x}+2^{-1}\left( \pm r_{1} h \boldsymbol{e}_{1}+r_{2} h \boldsymbol{e}_{2}\right)\right), \\
& a_{i j}^{ \pm-}(\boldsymbol{r})=a_{i j}\left(\boldsymbol{x}+2^{-1}\left( \pm r_{1} h \boldsymbol{e}_{1}-r_{2} h \boldsymbol{e}_{2}\right)\right),
\end{aligned}
$$

and $a_{i j}^{\alpha \beta}=a_{i j}^{\alpha \beta}(\mathbf{1})$ by convention. For instance, $a_{i j}^{++}(\boldsymbol{r})=a_{i j}\left(x^{(+)}(n, \boldsymbol{x}, \boldsymbol{r})\right), a_{i j}^{-+}(\boldsymbol{r})=$ $a_{i j}\left(x^{(+)}(n, \boldsymbol{x}, \boldsymbol{r})-r_{1} h \boldsymbol{e}_{1}\right)$, etc. Now we have the following result:

$$
\begin{aligned}
& \left(A_{n}\right)_{\boldsymbol{k} \boldsymbol{k} \pm \boldsymbol{e}_{1}}=-\frac{1}{h^{2}}\left(a_{11}^{ \pm+}-\frac{r_{1}}{r_{2}}\left|a_{12}^{ \pm+}(\boldsymbol{r})\right|\right), \\
& \left(A_{n}\right)_{\boldsymbol{k} \boldsymbol{k} \pm \boldsymbol{e}_{2}}=-\frac{1}{h^{2}}\left(a_{22}^{+ \pm}-\frac{r_{2}}{r_{1}}\left|a_{12}^{+ \pm}(\boldsymbol{r})\right|\right), \\
& \left(A_{n}\right)_{\boldsymbol{k} \boldsymbol{k} \pm\left(r_{1} \boldsymbol{e}_{1}-r_{2} \boldsymbol{e}_{2}\right)}=-\frac{1}{r_{1} r_{2} h^{2}} a_{12}^{ \pm \mp}(\boldsymbol{r}) .
\end{aligned}
$$


Obviously, the constructed schemes are analogs of the schemes (18) which are derived for the classical elliptic differential operator $A_{0}=-\sum_{i j} a_{i j} \partial_{i} \partial_{j}$. If $a_{12} \leq 0$ and

$$
\inf _{\boldsymbol{x} \in G_{n}}\left\{\frac{1}{r_{i} h} a_{i i}^{\alpha \beta}-\frac{1}{r_{j} h}\left|a_{12}^{\gamma \delta}(\boldsymbol{r})\right|\right\}>0, \quad \alpha, \beta, \gamma, \delta \in\{=,-\}
$$

the constructed matrix $A_{n}$ is compartmental. We call it the first extended scheme.

The second extended scheme can be constructed analogously starting from another bilinear form. Let us define $a_{n}^{(+)}(v, u)$ form $a_{n}^{(-)}(v, u)$ by the following simple change. Each $\Xi_{i}\left(r_{i} h\right)$ in (22) has to be replaced with $\Xi_{i}\left((-)^{i-1} h r_{i}\right)$. The obtained form is denoted by $a_{n}^{(+)}(v, u)$ and the corresponding system matrix $A_{n}$ has entries:

$$
\begin{aligned}
& \left(A_{n}\right)_{\boldsymbol{k} \boldsymbol{k} \pm \boldsymbol{e}_{1}}=-\frac{1}{h^{2}}\left(a_{11}^{ \pm-}-\frac{r_{1}}{r_{2}}\left|a_{12}^{ \pm-}(\boldsymbol{r})\right|\right), \\
& \left(A_{n}\right)_{\boldsymbol{k} \boldsymbol{k} \pm \boldsymbol{e}_{2}}=-\frac{1}{h^{2}}\left(a_{22}^{+ \pm}-\frac{r_{2}}{r_{1}}\left|a_{12}^{+ \pm}(\boldsymbol{r})\right|\right), \\
& \left(A_{n}\right)_{\boldsymbol{k} \boldsymbol{k} \pm\left(r_{1} \boldsymbol{e}_{1}+r_{2} \boldsymbol{e}_{2}\right)}=-\frac{1}{r_{1} r_{2} h^{2}} a_{12}^{ \pm \pm}(\boldsymbol{r}),
\end{aligned}
$$

in a complete analogy with (18). It is compartmental for $a_{12} \geq 0$.

It is important to point out that the schemes (23), (24) are obtained by a guess from the schemes (18), i.e. they are a priori defined from some general principle which is not based either on difference equations or variational equalities. Then bilinear forms (22) are constructed so that variational equalities are equivalent with the schemes. This non-conventional approach in the construction of schemes has an apparent drawback. The forms (22) do not seam to be positive definite. Actually, their positive definiteness can be ensured in the $\left.|\cdot|\right|_{\text {avg,2,1-norm, i.e. a weaker norm }}$ than $|\cdot|_{2,1}$-norm. This problem is analyzed in the next subsection.

In the case of a general function $a_{12}$ on $\mathbb{R}^{2}$ we cannot use only one of proposed two extended scheme. Here we give a construction of $A_{n}$, which discretize the differential operator $A_{0}$, possessing the compartmental structure. Our intention is to construct a sequence $a^{(r)}=\left\{a_{i j}^{(r)}\right\}_{11}^{22}$ of tensor-valued functions converging *-weakly in $L_{\infty}\left(\mathbb{R}^{2}\right)$ to the original tensor-valued function (11), such that:

1. Each $a^{(r)}$ defines a form $v, u \mapsto a^{(r)}(v, u)$ and for any fixed pair $v, u \in W_{2}^{1}\left(\mathbb{R}^{2}\right)$

$$
a(v, u)=\lim _{r} a^{(r)}(v, u) .
$$

2. The forms $a^{(r)}(\cdot, \cdot)$ can be discretized by using described extended schemes. The resulting discretizations are denoted by $h^{2} a_{n}^{(r)}(\cdot, \cdot)$.

3. The matrices $A_{n}^{(r)}$ defined by $a_{n}^{(r)}(v, u)=\left\langle\mathbf{v} \mid A_{n}^{(r)} \mathbf{u}\right\rangle$ have the compartmental structure.

In the first step of construction we assume that the functions $a_{i j}$ are uniformly continuous on $\mathbb{R}^{2}$. For each $r \in \mathbb{N}$ we define the sets

$$
\begin{aligned}
& F_{r}(+)=\left\{\boldsymbol{x} \in \mathbb{R}^{2}: a_{12}(\boldsymbol{x}) \geq r^{-1}\right\}, \\
& F_{r}(-)=\left\{\boldsymbol{x} \in \mathbb{R}^{2}: a_{12}(\boldsymbol{x}) \leq-r^{-1}\right\}, \quad r \in \mathbb{N} . \\
& F_{r}(0)=\mathbb{R}^{2} \backslash F_{r}(+) \cap F_{r}(-),
\end{aligned}
$$

Due to the uniform continuity of functions $a_{i j}$ there exists a positive $\varepsilon_{r}$ such that for sufficiently large $r$ and $n$ :

1. $\operatorname{dist}\left(F_{r}(+), F_{r}(-)\right)=\varepsilon_{r}>0$.

2. The set $F_{r}(0)$ has a finite number of connected components. 
3. To each connected component of $F_{r}(0)$ there corresponds one connected set of $F_{r}(0) \cap \operatorname{int}\left(\cup \boldsymbol{x} \in G_{n} C(h, \boldsymbol{x})\right)$.

Now we define the sets

$$
\begin{aligned}
& G_{n}^{( \pm)}=\cup_{\boldsymbol{x} \in F_{r}( \pm) \cap G_{n}} \overline{C(h, \boldsymbol{x})}, \\
& G_{n}^{(0)}=\mathbb{R}^{2} \backslash\left(G_{n}^{(+)} \cup G_{n}^{(-)}\right),
\end{aligned}
$$

and point out that for sufficiently large $n$ the sets $F_{r}(0)$ and $G_{n}^{(0)}$ have the same number of connected components. In addition, each connected component is equal to the union of interiors of certain cubes $C(h, \boldsymbol{x})$ with vertices $\boldsymbol{x} \in F_{r}(0)$. In order to complete a partition of $\mathbb{R}^{2}$ into subsets $G_{n}^{( \pm)}$we can extend the constructed sets $G_{n}^{( \pm)}$ in the following way. Each non-trivial $C(h, \boldsymbol{x}) \cap G_{n}^{(0)}$ can be attached to the nearest existing $G_{n}^{( \pm)}$. The first and second extended schemes have to be used for grid-knots in so completed $G_{n}^{( \pm)}$, respectively. The associated forms $a_{n}^{( \pm)}(v, u)$ are defined as in (22) with the following difference. Instead of the sum over grid-knots $\boldsymbol{x} \in G_{n}$, in the case of $a_{n}^{( \pm)}(v, u)$, we must have the sum over grid-knots in $G_{n}^{( \pm)}$, respectively. Then $a_{n}^{( \pm)}(v, u)=\left\langle\mathbf{v}_{n} \mid A_{n} \mathbf{u}_{n}\right\rangle$ and $A_{n}$ is compartmental. Of course, the parameter $\boldsymbol{r}$ varies on $G_{n}$ so appropriate notation is $\boldsymbol{r}(\boldsymbol{x})$.

In the last step of the construction we assume that the functions $a_{i j}$ are measurable. We have to smooth the functions $a_{i j}$ by a mollifier $\left\{\chi_{n}: n \in \mathbb{N}\right\}$, where $\chi_{n}(\boldsymbol{x})=n^{2} \chi_{1}(n \boldsymbol{x})$ and $\chi_{1}$ is a non-negative function with support in the disc $B_{1}(\mathbf{0})$. In this way we get a sequence of tensor-valued functions $a(n)=a * \chi_{n}$ converging $*$-weakly in $L_{\infty}\left(\mathbb{R}^{2}\right)$ to the original tensor-valued function $a$. The previous steps of constructions must be applied to the differential operators $A_{0}^{(r)}$ with coefficients $a_{i j}(n)$.

\subsection{Higher dimensions}

The index set of pairs $I(d)=\{\{i j\}: i<j, i, j=1,2, \ldots, d, i \neq j\}$ has the cardinal number $m(d)=d(d-1) / 2$. To each index $\{k l\} \in I(d)$ we associate three coefficients,

$$
a_{k k}^{\{k l\}}=\frac{1}{d-1} a_{k k}, \quad a_{l l}^{\{k l\}}=\frac{1}{d-1} a_{l l}, \quad a_{k l}^{\{k l\}}=a_{k l},
$$

and a bilinear form $a^{\{k l\}}(\cdot, \cdot)$,

$$
a^{\{k l\}}(v, u)=\sum_{i, j \in\{r, s\}} \int_{D} a_{i j}^{\{k l\}}(\boldsymbol{x}) \partial_{i} v(\boldsymbol{x}) \partial_{j} u(\boldsymbol{x}) d \boldsymbol{x} .
$$

Apparently, for each $v \in \dot{W}_{\infty}^{1}\left(\mathbb{R}^{d}\right), u \in \dot{W}_{1}^{1}\left(\mathbb{R}^{d}\right)$ with compact supports, the following equality is valid:

$$
a(v, u)=\sum_{\{k l\} \in I(d)} a^{\{k l\}}(v, u) .
$$

To each of the forms $a^{\{k l\}}(\cdot, \cdot)$ we can associate a sequence of forms $a_{n}^{\{k l\}}(\cdot, \cdot)$ and matrices $A_{n}^{\{k l\}}$ constructed by using extended schemes. Then the matrix

$$
A_{n}=\sum_{\{k l\} \in I} A_{n}^{\{k l\}}
$$

is a discretization of $A_{0}(\boldsymbol{x})$. In the construction of $A_{n}^{\{k l\}}$, pairs $\boldsymbol{r}^{\{k l\}}(\boldsymbol{x})=\left(r_{1}^{\{k l\}}(\boldsymbol{x}), r_{2}^{\{k l\}}\right)(\boldsymbol{x}) \in$ $\mathbb{N}^{2}$ can be used, generally depending on $\boldsymbol{x}$.

In the remaining part of this subsection the objects of analysis are the compartmental structure of (27) and strict ellipticity of forms $\mathbf{v}, \mathbf{u} \mapsto\left\langle\mathbf{v} \mid A_{n} \mathbf{u}\right\rangle$. 
For $\boldsymbol{x}=h \boldsymbol{k} \in G_{n}$ the neighborhoods $\mathcal{N}(\boldsymbol{x})$ have structures which are mutually similar one to the other. If all $a_{i j}(\boldsymbol{x}) \neq 0$ then the minimal number of elements in $\mathcal{N}(\boldsymbol{x})$ is $1+d+d^{2}$. In this case the set $\mathcal{N}(\boldsymbol{x})$ consists of its center, $2 d$-grid-knots on the $d$-dimensional cross $\left\{ \pm \boldsymbol{e}_{i}: i=1,2, \ldots, d\right\}$ and 2 grid-knots in each twodimension plane. Generally, the number of grid-knots in two-dimensional plane may be larger than 2. Here we consider only the case of at most two grid-knots in twodimensional planes. This demand has the following implication on the construction of discretizations $A_{n}^{(r s)}$. Let the pairs $\boldsymbol{e}_{r}, \boldsymbol{e}_{s}$ and $\boldsymbol{e}_{s}, \boldsymbol{e}_{t}$ define two-dimensional planes and let $A_{n}^{(r s)}, A_{n}^{(s t)}$ be the corresponding discretizations which are constructed by using parameters $\boldsymbol{r}^{(r s)}, \boldsymbol{r}^{(s t)}$. Then there must hold $\left(\boldsymbol{r}^{(r s)}\right)_{s}=\left(\boldsymbol{r}^{(s t)}\right)_{s}$. In such case the off-diagonal entries of $A_{n}$ have the following general structure:

$$
\begin{aligned}
& \left(A_{n}\right)_{\boldsymbol{k} \boldsymbol{k} \pm \boldsymbol{e}_{i}}=-\frac{1}{h^{2}}\left[\bar{a}_{i i}-\sum_{m \neq i} \frac{r_{i}}{r_{m}}\left|a_{i m}\left(\boldsymbol{x}_{i m}\right)\right|\right], \\
& \left(A_{n}\right)_{\boldsymbol{k} \boldsymbol{k} \pm \boldsymbol{m}_{i j}}=-\frac{1}{r_{i} r_{j} h^{2}}\left|a_{i j}\left(\boldsymbol{x}_{i j}\right)\right|,
\end{aligned}
$$

where $\bar{a}_{i i}$ are certain convex combinations of values $a_{i i}\left(\boldsymbol{x}_{i i}^{\{k l\}}\right), \boldsymbol{x}_{i j}^{\{k l\}}$ are certain points close to $\boldsymbol{x}$ which are defined by the extended schemes and $\boldsymbol{m}_{i j}$ is either $r_{i} \boldsymbol{e}_{i}+r_{j} \boldsymbol{e}_{j}$ or $r_{i} \boldsymbol{e}_{i}-r_{j} \boldsymbol{e}_{j}$, depending on the sign of $a_{i j}$.

Let us assume that the auxiliary tensor-valued function $\boldsymbol{x} \mapsto \hat{a}(\boldsymbol{x})=\left\{\hat{a}_{i j}(\boldsymbol{x})\right\}_{11}^{d d}$ fulfils the double inequality:

$$
\underline{M}(\hat{a})|\boldsymbol{z}|^{2} \leq \sum_{i, j} z_{i} \hat{a}_{i j}(\boldsymbol{x}) z_{j} \leq \bar{M}(\hat{a})|\boldsymbol{z}|^{2}, \quad \boldsymbol{x} \in \mathbb{R}^{d} .
$$

The following result is valid:

PROPOSITION 4.1 Let $a_{i j}$ be uniformly continuous on $\mathbb{R}^{d}$ and let the double inequality (29) be valid. There exist a function $\boldsymbol{x} \mapsto \boldsymbol{r}(\boldsymbol{x}) \in \mathbb{N}^{d}$, to be used in the construction of matrices $A_{n}^{\{k l\}}$, such that (27) has the compartmental structure.

In order to prove this proposition one has to apply Perron-Frobenius theorem to the matrix $\hat{a}(\boldsymbol{x})^{-1}$ and construct the eigenvector $\boldsymbol{z}(\boldsymbol{x})$, corresponding to the minimal eigenvalue of $\hat{a}(\boldsymbol{x})$, with the property $z_{i}(\boldsymbol{x}) \geq \underline{m}>0$, where $\underline{m}$ is $\boldsymbol{x}$-independent.

If the coefficients $a_{i j}$ are not continuous, we have to use a smoothing procedure and apply the derived results to smeared out coefficients $a_{i j}(n)=a_{i j} * \chi_{n}$.

Let us turn to the problem of strict ellipticity. A discrete form $a_{n}(\cdot, \cdot)$ on $l_{0}\left(G_{n}(R)\right) \times$ $l_{0}\left(G_{n}(R)\right)$ is said to be strictly elliptic $\mathrm{YO}$ if there exist two positive numbers $\underline{M}\left(a_{n}\right), \bar{M}\left(a_{n}\right)$ such that

$$
\underline{M}\left(a_{n}\right) \sum_{i=1}^{d}\left|U_{i}\left(r_{i}\right) \mathbf{u}\right|_{R 2}^{2} \leq\left\langle\mathbf{u} \mid A_{n} \mathbf{u}\right\rangle_{R} \leq \bar{M}\left(a_{n}\right) \sum_{i=1}^{d}\left|U_{i}\left(r_{i}\right) \mathbf{u}\right|_{R 2}^{2} .
$$

We shall say that a sequence of discrete forms $a_{n}(\cdot, \cdot)$ is strictly elliptic uniformly with respect to $n \in \mathbb{N}$ if the positive numbers $\underline{M}\left(a_{n}\right), \bar{M}\left(a_{n}\right)$ are independent of $n$.

The quantities $\bar{a}_{i i}$ in (28) have the general structure

$$
\begin{aligned}
\omega_{n}\left(a_{i i}, \boldsymbol{x}\right) & =\sum_{s=1}^{d} f_{s}(\boldsymbol{x}) a_{i i}\left(x_{i i}(n, \boldsymbol{x}, s)\right), \\
f_{s}(\boldsymbol{x}) & \geq 0, \quad \sum_{s=1}^{d} f_{s}(\boldsymbol{x})=1,
\end{aligned}
$$

where $\boldsymbol{x}_{i j}(n, \boldsymbol{x}, s)$ are defined by the construction of schemes.

PROPOSITION 4.2 Let $A_{0}=-\sum \partial_{i} a_{i j} \partial_{j}$ be discretized by matrices $A_{n}$ with the following properties:

a) Each $A_{n}$ has the compartmental structure whenever the double inequality (29) holds. 
b) There exist $p_{i}, t_{i} \in \mathbb{R}$ such that

$$
\left(A_{n}\right)_{\boldsymbol{k} \boldsymbol{k} \pm r_{i} \boldsymbol{e}_{i}}=-\frac{1}{h^{2}} p_{i}\left[\omega_{n}\left(a_{i i}, \boldsymbol{x}\right)-\sum_{m \neq i} t_{i}\left|a_{i m}\left(\boldsymbol{x}_{i m}(n, \boldsymbol{x})\right)\right|\right]
$$

at each grid-knot $\boldsymbol{x}=h \boldsymbol{k}$.

c) If $\boldsymbol{m}$ is not in the direction of $\pm \boldsymbol{e}_{i}, i=1,2, \ldots, d$, then $\left(A_{n}\right)_{\boldsymbol{k} \boldsymbol{k}+\boldsymbol{m}}$ does not depend on any of $a_{i i}, i=1,2, \ldots, d$.

Then the discrete forms $\mathbf{v}, \mathbf{u} \mapsto\left\langle\mathbf{v} \mid A_{n} \mathbf{u}\right\rangle_{R}$ are strictly elliptic on $l_{0}\left(G_{n}(R)\right) \times$ $l_{0}\left(G_{n}(R)\right)$ uniformly with respect to $n \in \mathbb{N}$.

Proof: Let us consider diffusion tensors $a, b$ where $a=\left\{a_{i j}\right\}_{11}^{d d}$ and $b$ is defined by $b_{i j}=a_{i j}-\kappa \delta_{i j}$. The corresponding auxiliary tensors are denoted by $\hat{a}, \hat{b}$ as usual. Due to the fact that $a, \hat{a}$ are positive definite uniformly with respect to the points of $\mathbb{R}^{d}$ there can be chosen $\kappa>0$ so that $b, \hat{b}$ are also positive definite on $\mathbb{R}^{d}$. Let us define matrices $H_{n}$ on $G_{n}(R)$ by the following non-trivial entries:

$$
\left(H_{n}\right)_{\boldsymbol{k} \boldsymbol{k}}=\frac{2 \kappa}{h^{2}} \sum_{i=1}^{d} \frac{1}{r_{i}^{2}}, \quad\left(H_{n}\right)_{\boldsymbol{k} \boldsymbol{k} \pm r_{i} \boldsymbol{e}_{i}}=-\frac{\kappa}{r_{i}^{2} h^{2}}, i=1,2, \ldots, d .
$$

We have $\left\langle\mathbf{v} \mid H_{n} \mathbf{u}\right\rangle_{R}=\kappa \sum_{i=1}^{d}\left|U_{i}\left(r_{i}\right) \mathbf{u}\right|_{R 2}^{2}$. The matrix $B_{n}=A_{n}-H_{n}$ has the compartmental structure in accordance with assumptions of this proposition. A matrix $B_{n}$ with the compartmental structure is necessarily positive definite, i.e. there must hold $\left\langle\mathbf{u} \mid B_{n} \mathbf{u}\right\rangle_{R} \geq 0$ for any $\mathbf{u} \in l_{0}\left(G_{n}(R)\right)$. Therefore

$$
\left\langle\mathbf{u} \mid A_{n} \mathbf{u}\right\rangle_{R}=\left\langle\mathbf{u} \mid H_{n} \mathbf{u}\right\rangle_{R}+\left\langle\mathbf{u} \mid B_{n} \mathbf{u}\right\rangle_{R} \geq\left\langle\mathbf{u} \mid H_{n} \mathbf{u}\right\rangle_{R}=\kappa \sum_{i=1}^{d}\left|U_{i}\left(r_{i}\right) \mathbf{u}\right|_{R 2}^{2},
$$

proving the assertion.

QED

The first order differential operators of (1) can be discretized by using upwinding method. In the case of continuous functions $b_{j}$ the resulting system matrices have the compartmental structure. If the coefficients $b_{j}$ are not continuous, the compartmental structure is obtained for sufficiently large $n$.

\section{Convergence of numerical solutions}

The difference schemes which are constructed in Section 4 enable us to generate gridsolutions $\mathbf{u}_{n} \in L\left(G_{n}(R)\right)$ and the corresponding imbedding $u(n)=\Phi_{n}(R) \mathbf{u}_{n}$ into $E_{n}\left(R, \mathbb{R}^{d}\right)$ spaces. Basic schemes are derived from variational equalities

$$
\left\langle\mathbf{v}_{n} \mid \lambda I+A_{n} \mathbf{u}_{n}\right\rangle=\left\langle\mathbf{v}_{n} \mid \boldsymbol{\mu}_{n}\right\rangle, \quad \mathbf{v}_{n} \in w_{2}^{1}\left(G_{n}\right),
$$

while extended schemes follow from a general principle which is not a priori related to any bilinear form and variational equalities. For a differential operators in divergence form $A_{0}$ the derived discretizations $A_{n}$ have the compartmental structure, i.e. they satisfy (17) only for constant functions. Consequently, as expected, we cannot use the result on convergence of Section 4. In order to prove the convergence of approximate solutions we must replace conditions of Lemma 4.1 with some other property. It is natural to look for a formulation in terms of variational equalities (30) and follow well known methods of finite elements. Unfortunately, we are faced with a difficulty. For extended schemes the bilinear form (22) does not seam to be strictly elliptic. The strict ellipticity of forms is a basic supposition in a proof of $W_{2}^{1}$-convergence. For this purpose we can use a general result of Proposition 4.2. The underlining idea is clear from analyzing the 2-dimensional problem with $a_{12} \leq 0$ on $\mathbb{R}^{2}$ and a fixed $\boldsymbol{r}=\left(r_{1}, r_{2}\right)$.

When Proposition 4.2 is applied to the present case we get 
COROLLARY 5.1 Let the auxiliary diffusion tensor $\hat{a}$ be strictly positive on $\mathbb{R}^{d}$. There exists a mapping $\boldsymbol{x} \mapsto \boldsymbol{r}(\boldsymbol{x}) \in \mathbb{N}^{d}$ such that the matrices $A_{n}$ of (27) have the compartmental structure, and the forms defined by $a_{n}(v, u)=\left\langle\mathbf{v} \mid A_{n} \mathbf{u}\right\rangle$ are strictly elliptic uniformly with respect to $n \in \mathbb{N}$.

For extended schemes Corollary 5.1 can be expressed simply as:

$$
\underline{M} \sum_{i=1}^{2}\left|U_{i} \mathbf{u}_{n}\right|_{2}^{2} \leq a_{n}(u, u) \leq \bar{M} \sum_{i=1}^{2}\left|U_{i} \mathbf{u}_{n}\right|_{2}^{2}
$$

with certain $\underline{M}, \bar{M}$. For basic schemes we must use averaged norms. In order to demonstrate that the averaged norms have no effect on the convergence of approximate solutions we proceed further by using averaged norms.

\subsection{Consistency}

For constant functions $a_{i j}$ we have an obvious equality:

$$
a(v, u)=\lim _{n} h^{d} a_{a v g, n}(\hat{v}(n), \hat{u}(n))
$$

implied by (ii) of Theorem 3.1 and Corollary 3.1. For non-constat functions $a_{i j}$ this property can be proved. Actually, we need a more general result.

PROPOSITION 5.1 (Consistency) Let $\mathfrak{V}=\{v(n): n \in \mathbb{N}\}$ and $\mathfrak{U}=\{u(n): n \in$ $\mathbb{N}\}, v(n), u(n) \in E_{n}\left(\mathbb{R}^{2}\right)$, converge weakly and strongly in $W_{2}^{1}\left(\mathbb{R}^{d}\right)$ to $v, u \in W_{2}^{1}\left(\mathbb{R}^{d}\right)$, respectively. Then

$$
a(v, u)=\lim _{n} h^{d}\left\langle\mathbf{v}_{n} \mid A_{n} \mathbf{u}_{n}\right\rangle .
$$

The present object of analysis is the functional $F_{n}(\cdot, \cdot)$ on $E_{n}\left(R, \mathbb{R}^{d}\right) \times E_{n}\left(R, \mathbb{R}^{d}\right)$, defined by

$$
F_{n}(v, u)=h^{d} \operatorname{vol}(R) \sum_{\boldsymbol{k} \in I_{n}(R)} p(\boldsymbol{x}(n, h \boldsymbol{k})) \square_{i}\left(r_{i} h\right) v(h \boldsymbol{k}) \Xi_{j}\left(r_{j} h\right) u(h \boldsymbol{k}),
$$

where $p$ is a bounded and uniformly continuous function on $\mathbb{R}^{d}, \boldsymbol{x}(n, \cdot)$ is a transformation of $\mathbb{R}^{d}$ such that $\left.\mid \boldsymbol{x}(n, h \boldsymbol{k})\right)-h \boldsymbol{k} \mid \leq \omega n^{-1}, \omega$ is fixed. Obviously, the form $h^{d} a_{n}(\cdot, \cdot)$ of (22) is a finite sum of $F_{a v g, n}$ with various $p=a_{i j}$. Therefore a proof of Proposition follows from the following result.

LEMMA 5.1 Let the sequence $\mathfrak{V}=\{v(n): n \in \mathbb{N}\} \subset \cup_{n} E_{n}\left(R, \mathbb{R}^{d}\right)$ converge $W_{2}^{1}$ weakly to $v \in W_{2}^{1}\left(\mathbb{R}^{d}\right)$ and the sequence $\mathfrak{U}=\{u(n): n \in \mathbb{N}\} \subset \cup_{n} E_{n}\left(R, \mathbb{R}^{d}\right)$ converge $W_{2}^{1}$-strongly to $u \in W_{2}^{1}\left(\mathbb{R}^{d}\right)$. Then

$$
\lim _{n} F_{n}(v(n), u(n))=\int p(\boldsymbol{x}) \frac{\partial v}{\partial x_{i}}(\boldsymbol{x}) \frac{\partial u}{\partial x_{j}}(\boldsymbol{x}) d \boldsymbol{x} .
$$

Proof: The integral on the right hand side is denoted by $P(v, u)$. We have to analyze $P(v(n), u(n))$ as $n \rightarrow \infty$. To simplify notation we assume $i, j \in\{1,2\}$. The indices $\boldsymbol{k}=\left(k_{1}, k_{2}, \ldots, k_{s}\right)$ are denoted shortly as $\boldsymbol{k}=\left(k_{1}, \boldsymbol{k}^{\prime}\right)$ and $\boldsymbol{k}=\left(k_{1}, k_{2}, \boldsymbol{k}^{\prime \prime}\right)$, $k_{1}, k_{2} \in \mathbb{Z}$. The corresponding $r_{1}, r_{2}$ are denoted by $r, s$, respectively. After inserting expressions for $v(n), u(n)$ and carrying out a straightforward calculation, we get the following expression for the case of $i=j=1$ :

$$
P(v(n), u(n))=\sum_{k, \boldsymbol{k}^{\prime}, \boldsymbol{l}^{\prime} \in I_{n}(R)} \int_{J(k, r)} d z\left(\psi_{\boldsymbol{k}^{\prime}} \mid p(z, \cdot) \psi_{\boldsymbol{l}^{\prime}}\right) v_{\left(k \boldsymbol{k}^{\prime}\right) i} u_{\left(k \boldsymbol{l}^{\prime}\right) i},
$$


where $v_{\boldsymbol{k} i}=\Xi_{i}\left(r_{i} h\right) v(h \boldsymbol{k}), u_{\boldsymbol{k} j}=\Xi_{j}\left(r_{j} h\right) u(h \boldsymbol{k})$ and $J(k, r)=[k h, k h+r h]$. Analogously we get for $i=1, j=2$ :

$$
\begin{gathered}
P(v(n), u(n))=\sum_{k, l, \boldsymbol{k}^{\prime \prime}, l^{\prime \prime} \in I_{n}(R)} \int_{J(k, r) \times J(l, s)} d z_{1} d z_{2}\left(\psi_{\boldsymbol{k}^{\prime \prime}} \mid p\left(z_{1}, z_{2}, \cdot\right) \psi_{\boldsymbol{l}^{\prime \prime}}\right) \\
\times\left[v_{\left(k, l, \boldsymbol{k}^{\prime \prime}\right) i} u_{\left(k, l, l^{\prime \prime}\right) j} \psi_{k}\left(z_{1}\right) \psi_{l}\left(z_{2}\right)+v_{\left(k, l, \boldsymbol{k}^{\prime \prime}\right) i} u_{\left(k+r, l, \boldsymbol{l}^{\prime \prime}\right) j} \psi_{k+r}\left(z_{1}\right) \psi_{l}\left(z_{2}\right)\right. \\
\left.+v_{\left(k, l+s, \boldsymbol{k}^{\prime \prime}\right) i} u_{\left(k, l, l^{\prime \prime}\right) j} \psi_{k}\left(z_{1}\right) \psi_{l+s}\left(z_{2}\right)+v_{\left(k, l+s, \boldsymbol{k}^{\prime \prime}\right) i} u_{\left(k+r, l, l^{\prime \prime}\right) j} \psi_{k+r}\left(z_{1}\right) \psi_{l+s}\left(z_{2}\right)\right] .
\end{gathered}
$$

Now we approximate the obtained expressions by inserting specific values of $p$ in the integrals over intervals $J(k, r)$. The error arised from approximation can be estimated by a bound depending on the product of $\operatorname{osc}(p, n)=\sup \{|p(\boldsymbol{x})-p(\boldsymbol{y})|$ : $\left.\boldsymbol{x}, \boldsymbol{y} \in \mathbb{R}^{d},\left|x_{i}-y_{i}\right| \leq 4 r_{i} h\right\}$ and the norms $\left|U_{i}\left(r_{i}\right) \mathbf{v}\right| \mathbf{|}_{R 2}, \mid U_{j}\left(r_{j}\right) \mathbf{u} \mathbf{|}_{R 2}$. For instance, for $i=j=1$ the resulting expressing is

$$
Q_{n}(v(n), u(n))=h^{d} \operatorname{vol}(R) \sum_{k, \boldsymbol{k}^{\prime}, \boldsymbol{l}^{\prime} \in I_{n}(R)} p(\boldsymbol{x}(n, h \boldsymbol{k})) s_{\boldsymbol{k}^{\prime} \boldsymbol{l}^{\prime}} v_{\left(k, \boldsymbol{k}^{\prime}\right) i} u_{\left(k, \boldsymbol{l}^{\prime}\right) j},
$$

where generally $s_{\boldsymbol{k} \boldsymbol{l}}=\left(\psi_{\boldsymbol{k}} \mid \psi_{\boldsymbol{l}}\right)\left\|\psi_{\boldsymbol{k}}\right\|_{1}^{-1}$. The quantities $s_{\boldsymbol{k} \boldsymbol{l}}$ have properties $s_{\boldsymbol{k} \boldsymbol{l}} \geq$ $0, \sum_{l} s_{k l}=1$. In addition

$$
\begin{gathered}
\left|P(v(n), u(n))-Q_{n}(v(n), u(n))\right| \leq \operatorname{osc}(p, n) h^{d}|\mathbf{v}|_{R 2}|\mathbf{u}|_{R 2} \\
\leq \operatorname{osc}(p, n)\left(1-\sigma^{2}\right)^{-1}\|v(n)\|_{2,1}\|u(n)\|_{2,1} .
\end{gathered}
$$

Instead of $P(v(n), u(n))$ we consider in the following $Q_{n}(v(n), u(n))$. The quantity $Q_{n}$ would be equal $F_{n}$ if $s_{\boldsymbol{k} \boldsymbol{l}}$ were absent and the double sum were replaced with the single sum over indices $\boldsymbol{k}$.

$Q_{n}(v(n), u(n))-F_{n}(v(n), u(n))=h^{d} \operatorname{vol}(R) \sum_{\boldsymbol{k} \boldsymbol{l}} p(\boldsymbol{x}(n, h \boldsymbol{k})) v_{\boldsymbol{k} i} \delta_{k \boldsymbol{l}} s_{\boldsymbol{k}^{\prime} \boldsymbol{l}^{\prime}}\left(u_{\boldsymbol{l} j}-u_{\boldsymbol{k} j}\right)$.

To estimate the right hand side we need $\bar{p}=\sup p$.

$$
\begin{gathered}
\left|Q_{n}(v(n), u(n))-F_{n}(v(n), u(n))\right| \leq \\
\bar{p} h^{d / 2}\left|U_{i}\left(r_{i}\right) \mathbf{v}_{n}\right| \begin{array}{l}
R 2 \\
h^{d / 2} \max \left\{\left|\left(Z\left(r_{j}, j\right)-I\right) U_{j}\left(r_{j}\right) \mathbf{u}_{n}\right|_{R 2}: j=1,2, \ldots, d\right\} .
\end{array}
\end{gathered}
$$

Due to the strong convergence of $\mathfrak{U}$, Lemma 3.3 and Corollary 3.1 we have for $\boldsymbol{w}=$ $h r_{j} \boldsymbol{e}_{j}$ :

$$
h^{d / 2} \mathbf{|}\left(Z\left(r_{j}, j\right)-I\right) U_{j}\left(r_{j}\right) \mathbf{u}_{n} \mathbf{\}_{R 2} \leq\left(1-\sigma^{2}\right)^{-1 / 2}\|(Z(\boldsymbol{w})-I) u(n)\|_{2}
$$

so that $\lim _{n} F_{n}(v(n), u(n))=\lim _{n} P(v(n), u(n))=P(v, u)$.

QED

\section{$5.2 W_{2}^{1}$-convergence}

It is described until now how the form $\lambda(v \mid u)+a(v, u)$ is discretized by forms $h^{d} \lambda\left\langle\mathbf{v}_{n} \mid \mathbf{u}_{n}\right\rangle+$ $\left\langle\mathbf{v}_{n} \mid A_{n} \mathbf{u}_{n}\right\rangle$. In order to solve the discretized problem (9) or (30), we have to describe how to discretize the linear function $v \rightarrow\langle v \mid \mu\rangle$ by $h^{d}\left\langle\mathbf{v}_{n} \mid \boldsymbol{\mu}_{n}\right\rangle$, where $\boldsymbol{\mu}_{n} \in l\left(G_{n}(R)\right)$. First we have to demonstrate the existence of $\boldsymbol{\mu}_{n}$ such that $h^{d}\left\langle\mathbf{v}_{n} \mid \boldsymbol{\mu}_{n}\right\rangle \rightarrow\langle v \mid \mu\rangle$.

LEMMA 5.2 Let $\mu$ be a continuous linear functional on $W_{2}^{1}\left(\mathbb{R}^{d}\right)$. There exists discretizations $\boldsymbol{\mu}_{n}(R) \in l\left(G_{n}(R)\right)$ such that

$$
\lim _{n}\left|\frac{h^{d}\left\langle\mathbf{u}_{n}(R) \mid \boldsymbol{\mu}_{n}(R)\right\rangle_{R}-\langle u(R, n) \mid \mu\rangle}{\|u(R, n)\|_{2,1}}\right|=0
$$

for any $R$. 
Proof: It suffices to consider the case $\mu=\partial f, f \in L_{2}\left(\mathbb{R}^{d}\right),\|\mu\|_{2,-1}=\|f\|_{2}$. Let us define

$$
\left(\boldsymbol{\mu}_{n}\right)_{\boldsymbol{k}}=-h^{-d}\left(\partial \psi_{\boldsymbol{k}} \mid f\right) \text {. }
$$

By a straightforward calculation we get

$$
\langle u(R, n) \mid \mu\rangle=\sum_{\boldsymbol{k}} u_{\boldsymbol{k}}\left\langle\psi_{\boldsymbol{k}} \mid \partial f\right\rangle=h^{d} \sum_{\boldsymbol{k}} u_{\boldsymbol{k}} \mu_{\boldsymbol{k}}=h^{d}\left\langle\mathbf{u} \mid \boldsymbol{\mu}_{n}\right\rangle_{R},
$$

proving Assertion.

QED

In other words the sequence of functions $\mu(n)=\Phi_{n}(R) \boldsymbol{\mu}_{n}(R) \in E_{n}\left(R, \mathbb{R}^{d}\right)$ converges strongly in $W_{2}^{-1}\left(\mathbb{R}^{d}\right)$ to $\mu$. This property implies the convergence of numbers $\langle v(n) \mid \mu(n)\rangle$ to the number $\langle v \mid \mu\rangle$ for any $W_{2}^{1}$-weakly convergent sequence of functions $v(n)$.

From the expression in proof of this Lemma we can get the estimate

$\left|\left\langle\mathbf{u}_{n} \mid \boldsymbol{\mu}_{n}\right\rangle_{R}\right|=h^{-d}|(\partial u(R, n) \mid f)| \leq h^{-d}\|\mu\|_{2,-1}\|\partial u(R, n)\|_{2} \leq h^{-d / 2}\|\mu\|_{2,-1} q_{R}\left(\mathbf{u}_{n}\right)^{1 / 2}$,

where the last inequality follows from Theorem 3.1. The obtained inequality is valid for any $R$, implying:

$$
\left|\boldsymbol{\mu}_{n}\right|_{\text {avg }, 2,-1}=\frac{1}{\operatorname{vol}(R)} \sum_{R}\left|\boldsymbol{\mu}_{n}\right|_{R 2,-1} \leq h^{-d / 2}\|\mu\|_{2,-1} .
$$

Inequality (32) and the variational equalities (30) imply the first result towards our proof of convergence of approximate solutions. If $\mathbf{u}_{n}$ solve (30) then

$$
\underline{M}\left|\mathbf{u}_{n}\right|_{\text {avg }, 2,1}^{2} \leq\left\langle\mathbf{u}_{n} \mid\left(\lambda I+A_{n}\right) \mathbf{u}_{n}\right\rangle \leq\left|\mathbf{u}_{n}\right|_{\text {avg }, 2,1}\left|\boldsymbol{\mu}_{n}\right|_{\text {avg }, 2,-1} .
$$

COROLLARY 5.2 Let $\mathbf{u}_{n}=R\left(\lambda, A_{n}\right) \boldsymbol{\mu}_{n}$ and $u(R, n)=\Phi_{n}(R) \mathbf{u}_{n}$. Then for each $R$ the sequence $\mathfrak{U}=\{u(R, n): n \in \mathbb{N}\} \subset \cup_{n} E_{n}\left(R, \mathbb{R}^{d}\right)$ converges weakly in $W_{2}^{1}\left(\mathbb{R}^{d}\right)$ to some $u \in W_{2}^{1}\left(\mathbb{R}^{d}\right)$.

Let $u^{*}$ be the solution of (5). Then the sequence of functions $\hat{u}^{*}(n)$, defined by (14), strongly converges to $u^{*}$ in $W_{2}^{1}$. In the remaining part of this analysis we have to demonstrate the expected property $\lim _{n} u(R, n)=\lim _{n} \hat{u}^{*}(R, n)=u^{*}$ for each $R$. We follow the well-known finite element technique.

$$
\begin{aligned}
\underline{M} h^{d}\left|\mathbf{u}_{n}-\hat{\mathbf{u}}_{n}^{*}\right|_{\text {avg,2,1 }}^{2} & \leq h^{d}\left\langle\mathbf{u}_{n}-\hat{\mathbf{u}}_{n}^{*} \mid\left(\lambda I+A_{n}\right)\left(\mathbf{u}_{n}-\hat{\mathbf{u}}_{n}^{*}\right)\right\rangle \\
& =h^{d}\left\langle\mathbf{u}_{n}-\hat{\mathbf{u}}_{n}^{*} \mid\left(\lambda I+A_{n}\right) \mathbf{u}_{n}\right\rangle \\
& -h^{d}\left\langle\mathbf{u}_{n}-\hat{\mathbf{u}}_{n}^{*} \mid\left(\lambda I+A_{n}\right) \hat{\mathbf{u}}_{n}^{*}\right\rangle \\
=h^{d}\left\langle\mathbf{u}_{n}-\hat{\mathbf{u}}_{n}^{*} \mid \boldsymbol{\mu}_{n}\right\rangle & -h^{d}\left\langle\mathbf{u}_{n}-\hat{\mathbf{u}}_{n}^{*} \mid\left(\lambda I+A_{n}\right) \hat{\mathbf{u}}_{n}^{*}\right\rangle .
\end{aligned}
$$

By Lemma 5.2 the first term on the right hand side converges to $\left\langle u-u^{*} \mid \mu\right\rangle$. By the consistency property of Proposition 5.1 the second term converges to the same value.

THEOREM 5.1 Let $\mathfrak{U}$ be as in Corollary 5.2. Then the sequence $\mathfrak{U}$ converges $W_{2}^{1}(\mathbb{R})$-strongly to the unique solution $u^{*}$ of (5).

From this result, Lemma 5.2 and Lemma 3.2 we get another important result for $\lambda=0$.

COROLLARY 5.3 Let $D$ be a bounded domain with Lipsithz boundary and $\mu \in$ $\dot{W}_{2}^{-1}(D)$. Let $A_{n}(D)$ be the restriction to $G_{n}(D)$ of $A_{n}, \boldsymbol{\mu}_{n}$ on $G_{n}(D)$ satisfy (31) and $\mathbf{u}_{n}=A_{n}(D)^{-1} \boldsymbol{\mu}_{n}$. Then the sequence $\mathfrak{U}$ converges strongly in $W_{2}^{1}\left(\mathbb{R}^{d}\right)$ to the unique weak solution $u$ of (5). 


\subsection{Convergence in $C$ - and $L_{1}$-spaces}

Regularity properties of solutions of the converging sequences $\mathfrak{U}$ of Corollary 5.3 follow from the well-known results of DeGiorgi type. We can use criteria developed by [LU] as in our approach [LR3] in order to get the following result. If $\|\mu\|_{\infty}=1$ then there exists $\alpha>0$ such that $\mathfrak{U}$ of Corollary 5.3 converges in the Hölder spaces $C^{(\alpha)}(\bar{D})$ to $u$ of (5). Finally, if $\mu \in L_{1}(D)$, or $\mu \in \mathcal{P}(D)$, numerical solutions must converge in the Banach space $\dot{L}^{(\alpha)}(D)$, which is defined in [St]. This space is the completion of $\dot{C}^{(\alpha)}(\bar{D})$ in the norm:

$$
\|u\|=\|u\|_{1}+\sup _{|\boldsymbol{w}|>0} \frac{1}{|\boldsymbol{w}|^{\alpha}}\|(Z(\boldsymbol{w})-I) u\|_{1} .
$$

Due to the fact that the spaces $E_{n}\left(D, \mathbb{R}^{d}\right)$ are finite-dimensional, we can use the duality of finite-dimensional spaces $l_{1}\left(G_{n}(D)\right)$ and $l_{\infty}\left(G_{n}(D)\right)$ and get a result on the convergence in $L_{1}(D)$-space. In order to describe the convergence in Hölder space and $L_{1}(D)$-space we have to explain some details about discretizations. For the discussion of convergence in Hölder space we assume $\mu=f \in L_{\infty}(D),\|f\|_{\infty}=1$, while for the convergence in $L_{1}(D)$-space we assume $\mu \in \mathcal{P}(D)$. Discretizations of $f$ are denoted by $\mathbf{f}_{n}$ and it is supposed that the sequence of functions $f(n)=\Phi \mathbf{f}_{n}$ converges strongly to $f$ in $L_{2}(D)$. The grid solutions $\mathbf{u}_{n}=A_{n}^{-1} \mathbf{f}_{n}$ define a sequence of approximate solutions $\mathfrak{U}=\{u(n): n \in \mathbb{N}\} \subset \cup_{n} E_{n}(D)$. Discretizations of $\mu \in \mathcal{P}(D)$ are denoted by $\boldsymbol{\mu}_{n}$ and constructed so that $\mu(n) \in L_{1}(D), \lim _{n}\|\mu(n)\|_{1}=1$, as well as $\lim _{n}\langle g \mid \mu(n)\rangle=\langle g \mid \mu\rangle$ for each $g \in \dot{C}(D)$. The corresponding grid-solutions $\mathbf{v}_{n}=A_{n}^{-1} \boldsymbol{\mu}_{n}$ define a sequence of approximate solutions $\mathfrak{V}=\{v(n): n \in \mathbb{N}\} \subset \cup_{n} E_{n}(D)$.

COROLLARY 5.4 The sequence $\mathfrak{U}$ has a subsequence converging to $u$ in $\dot{C}^{(\alpha)}(\bar{D})$ for some $\alpha>0$. The sequence $\mathfrak{V}$ has a subsequence converging to $u$ in $L_{1}^{(\beta)}(D)$ for some $\beta>0$.

\section{Numerical realizations}

The convergence proofs of previous section were given for a simple case of only one $r \in \mathbb{N}^{d}$ realizing the compartmental structure of system matrices. In applications the coefficients $a_{i j}, i \neq j$ vary and we can gain the compartmental structure by using several parameters $\boldsymbol{r}$. The set $\mathbb{R}^{d}$ as well as $D \subset \mathbb{R}^{d}$ must be decomposed into subsets $D_{l}$ which are defined during description of basic schemes. It is assumed that the compartmental structure of system matrices $A_{n}$ can be attained by a single parameter $\boldsymbol{r}(l)$ for each $G_{n}(l)=G_{n} \cap \overline{D_{l}}$. Because of a numerous technical details, we cannot go into a thorough analysis of the construction of system matrices for variable $\boldsymbol{r}$. Nevertheless, in the following example this approach is undertaken, and seems to be natural.

\section{EXAMPLE 6.1}

In this example we consider the differential operator $A=-\sum_{i j=1}^{2} \partial_{i} a_{i j} \partial_{j}$ with the diffusion tensor defined as follows

$$
a=\left[\begin{array}{cc}
\sigma^{2} & \alpha(\boldsymbol{x}) \\
\alpha(\boldsymbol{x}) & 1
\end{array}\right], \quad \alpha(\boldsymbol{x})=\rho \mathbb{1}_{D_{0}}(\boldsymbol{x}), \quad \rho^{2}<\sigma^{2},
$$

where $\sigma^{2}$ is a positive number, $\rho$ is a real number and $D_{0}=(1 / 4,3 / 4)^{2}$.

Let $D=(0,1)^{2} \subset \mathbb{R}^{2}$ and $\partial D$ be its boundary. The function $\boldsymbol{x} \mapsto u^{*}\left(x_{1}, x_{2}\right)=$ $x_{1} x_{2}$ is the unique solution to the following boundary value problem

$$
\begin{aligned}
& (A u)(\boldsymbol{x})=f(\boldsymbol{x}) \quad \text { for } \quad \boldsymbol{x} \in D, \\
& u\left|\partial D=u^{*}\right| \partial D,
\end{aligned}
$$


where

$$
f(\boldsymbol{x})=2 \rho \mathbb{1}_{D_{0}}(\boldsymbol{x})+\frac{\rho}{4}\left[\delta\left(x_{1}-\frac{1}{4}\right)-3 \delta\left(x_{1}-\frac{3}{4}\right)+\delta\left(x_{2}-\frac{1}{4}\right)-3 \delta\left(x_{2}-\frac{3}{4}\right)\right] .
$$

The set $\mathbb{R}^{2}$ is discretized by the grid $G_{n}$ of grid-knots $\boldsymbol{x}_{k l}=h k \boldsymbol{e}_{1}+h l \boldsymbol{e}_{2}, k, l \in \mathbb{Z}$, where $h$ is a grid-step. In order to get a discretization of $D$ suitable for numerical handling we assume $h=1 / N$ where $N=4 M$. In this way we have discretizations $G_{n}(D)$ of the open square $D=(0,1)^{2}$ defined by grid-knots $\boldsymbol{x}_{k l}=\left(x_{k}, y_{l}\right), 1 \leq k, l<N$. The sets $G_{n}(2)=\overline{D_{0}} \cap G_{n}$ and $G_{n}(1)=G_{n} \backslash G_{n}(0)$ define a partition of $G_{n}$. The set $G_{n}(2)$ is closed, while $G_{n}(1)$ is open. Let $G_{n}(1, D)=G_{n}(1) \cap D$. Then $G_{n}(2), G_{n}(1, D)$ is a partition of $G_{n}(D)$ to be used in constructions of numerical grids and approximate solutions.

Figure 6.1

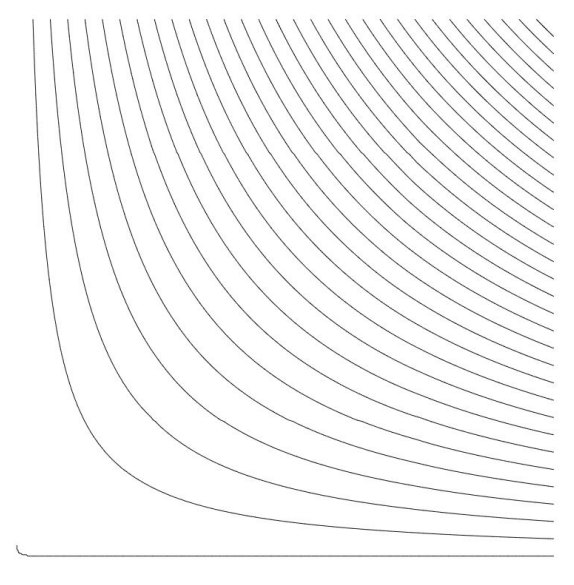

To demonstrate the efficiency of the extended schemes we choose $\sigma^{2}=10, \rho=2$ and the scheme parameters $r_{1}=1, r_{2}=3$ as illustrated in Figure 4.1 part (c). These values of parameters ensure the compartmental structure of the system matrix.

For a numerical illustration we discretize the domain $D$ by $399 \times 399$ grid-knots with the grid-step $h=1 / 400$, and define the grid-solution by the linear system (9). This system is then solved by iterations as follows. Let $K_{n}=\operatorname{diag}\left(A_{n}\right)$ and $Q_{n}=$ $A_{n}-\operatorname{diag}\left(A_{n}\right)$. Then $Q_{n} \geq 0$ and

$$
A_{n}^{-1}=\sum_{k=0}^{\infty}\left(K_{n}^{-1} Q_{n}\right)^{k} K_{n}^{-1} .
$$

Let $\mathbf{u}_{n}(r)$ be the approximation of $\mathbf{u}_{n}$ after $r$ iterations. By taking the stopping criteria to be $\left.\left|\mathbf{u}_{n}(r+1)-\mathbf{u}_{n}(r)\right|\right|_{1}<10^{-9}$, we have found that the iteration terminates after $r=210$ iterations. Then we compared the numerical approximations $\mathbf{u}_{n}(210)$ and values of the solution in $L_{\infty}(D)$ and $L_{1}(D)$-norms. The $L_{\infty}(D)$-norm of the difference $u(h \boldsymbol{k})-\left(\mathbf{u}_{n}(210)\right)_{\boldsymbol{k}}$ was 0.004 , realized at the grid-knot with coordinates $(0.75,0.75)$ which is one of the discontinuity points for $a_{12}$. The relative error in $L_{1}(D)$-norm was estimated by

$$
\varepsilon_{r e l}=100 \frac{\mid \mathbf{u}^{*}-\mathbf{u}_{n}(210) \boldsymbol{|}_{1}}{\left|\mathbf{u}^{*}\right|_{1}}
$$

where $\left(\mathbf{u}^{*}\right)_{\boldsymbol{k}}=u(h \boldsymbol{k})$. We obtained $\varepsilon_{r e l}=0.0008$, and the corresponding approximate solution $u(n)$ as illustrated in the Figure 6.1. 


\section{References}

[BS] G. Barles and P. E. Sougandis, Convergence of approximation schemes for fully nonlinear second order equations Asymptotic Analysis Vol 5., 271-283, 1991

[GT] D. Gilberg and N. S. Trudinger, Elliptic Partial Differential Equations, Springer, Berlin, 1983

[LU] O. A. Ladyzhenskaya and N. N. Ural'tseva, Linear and Quasilinear Elliptic Partial Differential Equations, Academic Press, N.Y., 1968

[LR1] N. Limić AND M. Rogina, Explicit stable methods for second order parabolic systems, Math. Commun. Vol 5, 97-115, 2000

[LR2] N. Limić And M. Rogina, Numerical solutions of Dirichlet problem for elliptic operator in divergence form with a right-hand side measure, J. Comput. Appl. Math., 493-516, 2004

[LR3] N. Limić And M. Rogina, Numerical approach to $L_{1}$-problems with the second order elliptic operators, preprint.

[MW] T. S. Motzkyn And W. Wasov, On the approximation of linear elliptic differential equations by difference equations with positive coefficients, J. Math. Phys. 31, 253-259, 1953

[Ma] V. G. MaZ'Ya, Sobolev spaces, Springer, N.Y., 1985

[RM] R. D. Richtmyer and K. W. Morton, Difference Methods for Initial-Value Problems, 2nd ed., Interscience, New York, 1967

[SMMM $]$ A. A. Samarskit, P. P. Matus, V. I. Mazhukin and I. E. Mozolevski, Monotone Difference Schemes for Equations with Mixed Derivatives, Comput. Math. Appl. 44, 501-510, 2002

[St] F. M. Stein, Singular Integrals and Differentiability Properties of Functions, Princeton Univ. Press, New Jersey, 1970

[Yo] D. M. Young, Iterative Methods for Solving Partial Difference Equations of Elliptic Type, thesis, Harvard University, Cambridge, Mass, 1950 